\title{
TRANSPA: A Code for Transient Thermal Analysis of a Single Fuel Pin
}

\author{
F. Coyne Prenger
}

\section{DISCLAIMER}

\begin{abstract}
This report was prepared as an account of work sponsored by an agency of the United States Government. Neither the United States Government nor any agency thereof, nor any of their employees, makes any warranty, express or implied, or assumes any legal uability or responsibility for the accuracy, completeness, or usefulness of any information, apparatus, product, or process disclosed, or represents that its use would not infringe privately owned rights. Reference herein to any specific commercial product, process, or service by trade name, trademark, manufacturer, or otherwise does not necessarily constitute or imply its endorsement, recommendation, or favoring by the United States Government or any agency thereof. The views and opinions of authors expressed herein do not necessarily state or reflect those of the United States Government or any agency thereot:
\end{abstract}
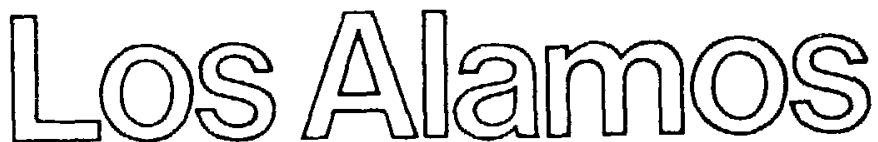
TRANSPA: A CODE FOR TRANSIENT THERMAL ANALYSIS OF A SINGLE FUEL PIN

by

F. Coyne Prenger

\begin{abstract}
An analytical model (TRANSPA) for the transient thermal analysis of a single uranium carbide fuel pin was developed. This model uses thermal boundary conditions obtained from COBRA-WC output and calculates the transient thermal response of a single fuel pin to changes in internal power generation, coolant flowrate, or fuel pin physical configuration. The model uses the MITAS finite difference thermal analyzer. MITAS provides the means to input separate conductance models through the use of a user subroutine input capability. The model is a lumped-mass representation of the fuel pin using 26 nodes and 42 conduciors. Run time for each transient analysis is approximately one minute of central processor time on the NOS operating system.
\end{abstract}

\title{
I. INTRODUCTION
}

The Transient Single Pin Analysis (TRANSPA) model uses the finite difference thermal analyzer code MITAS $^{1}$ as its basis. MITAS uses a Tumped-mass thermal network and can accept user written subroutines describing physical processes of interest. In addition MITAS contains an extensive library of subroutines that includes network solutions for both transient and steady state analyses.

TRANSPA is a thermal model of a single uranium-plutonium LMFBR carbide fuel pin located within a fuel pin assembly. Its primary purpose is to provide cladding temperature histories for selected transient events such as transient 
overpowers, loss of coolant simulations, or control rod transients. These cladding temperatures are used as input to the SPECKEL and ABAQUS ${ }^{2}$ code that is used to assess fuel performance. Boundary conditions for TRANSPA are obtained from the results of a complete assembly analysis of the fuel pin bundle. At present these results are obtained using the COBRA-WC ${ }^{3}$ thermal-hydraulics code. The procedure is to determine the steady state temperatures and flow distribution of the complete pin bundle using COBRA-WC. From these results the hot fuel pin is identified. The transient response of the hot pin or other selected pin is then analyzed using TRANSPA with flowrate and inlet temperature obtained from the COBRA-WC analysis. A description of the TRANSPA model follows.

\section{MODEL DESCRIPTION}

TRANSPA is a network thermal model of a single fuel pin. Boundary conditions are coolant flowrate and inlet temperature. The heat source is uniform internal heat generation that is specified by the pin location and time in the reactor. A description of the thermal network is given followed by discussion of the special subroutines, the input power profile and the network solution characteristics.

\section{A. Thermal Network}

The thermal network comprises 26 nodes and 42 conductors. The fuel pin is divided into five axial stations each of length $18.3 \mathrm{~cm}$. Each axial segment is divided into 4 radial segments representing the fuel centerline, the fuel surface, the clad inner surface and the clad outer surface. In addition an inlet and outlet fluid node is specified for each segment. The heat generation is simulated by a source term for the fuel centerline at each segment and the heat sink is a specified coolant inlet temperature at the bottom of the fuel pin. Both radiation and conduction are used to calculate the radial heat transfer across the fuel-cladding gap. Axial conduction in the fuel and cladding is also included. The convective heat transfer coefficient is used to calculate a heat transfer effectiveness that is used to calculate thermal couplings between the coolant and the fuel cladding. The thermal neiwork is shown in Fig. 1. 


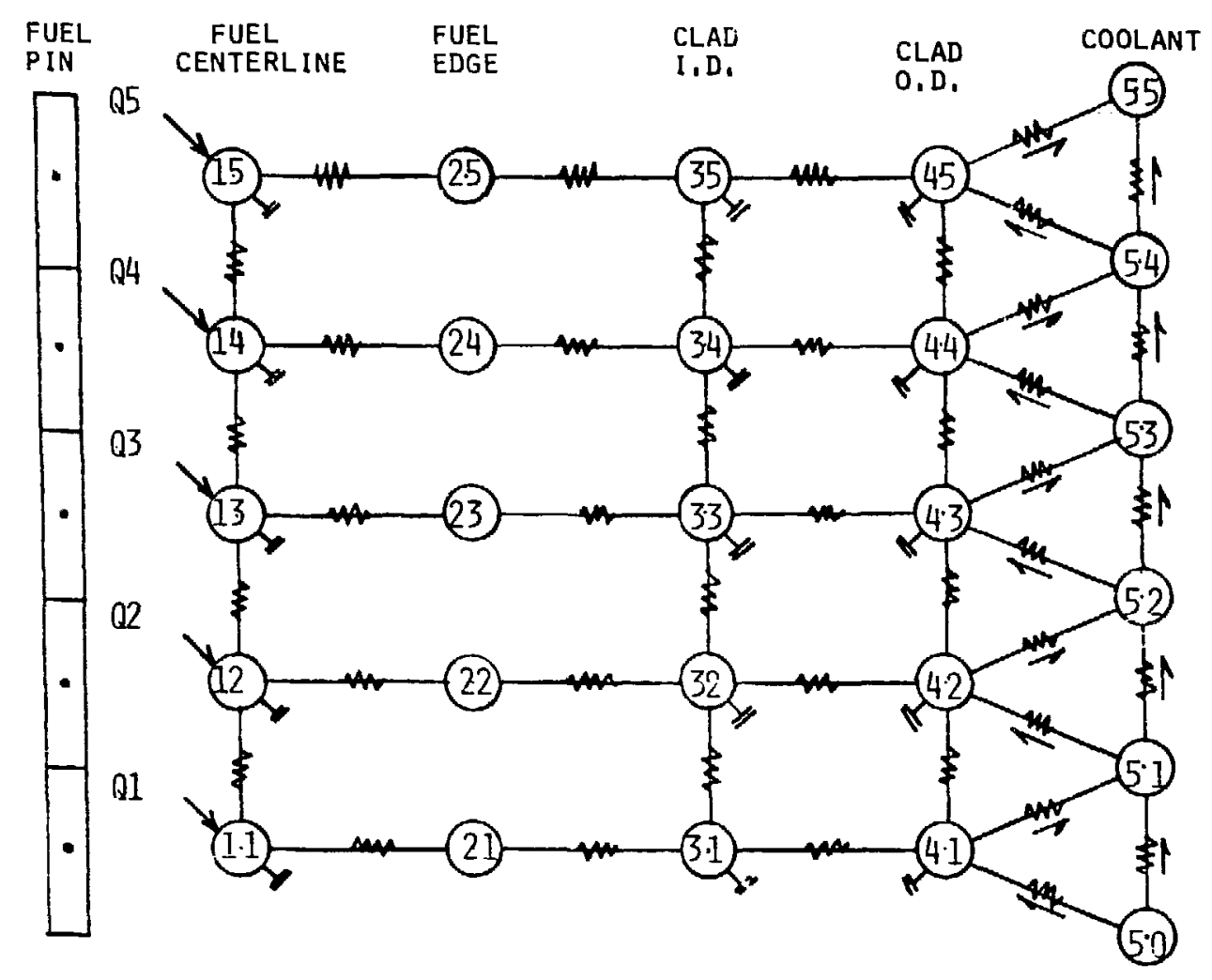

Fig. 1. Thermal network.

The thermal time constant of the model is small requiring small transient time steps. Also abrupt physical changes in the configuration lead to numerical instability in the model. These characteristics are discussed in a separate section. User subroutines required for the thermal network are discussed next.

B. Subroutines

User written subroutines can be easily incorporated into the MITAS code. Three subroutines are required for the TRANSPA model. The first subroutine calculates the gap conductance between the fuel and cladding. The gap conductance is a function of the interface pressure between the fuel and cladding. The subroutine uses initial conditions from either SPECKLE III or LIFE-4C code and then calculates changing interface pressure based on temperature dependent coefficients of themal expansion and moduli of elasticity. The routine cannot currently handle plasticity. The interface pressure is then 
used to approximate the gap conductance based on a curve fit generated with data predicted by the LIFE-4C code.

The second subroutine calculates the convective heat transfer coefficient of the coolant. This subroutine uses empirical data that is a function of the Reynolds and Prandtl numbers. ${ }^{3}$ The convective coefficient is then used to calculate a heat exchanger effectiveness that is used to specify the thermal couplings.

The final subroutine calculates the fuel conductivity as a function of temperature and porosity. ${ }^{4}$ Each of the subroutines represents a separate thermal conductance model based on either empirical data or results from a separate analysis. The structure of the MITAS code facilitates the parameterization of the TRANSPA model through the use of these user supplied subroutines.

\section{Power Profiles}

The heat source term is imposed on the fuel centerline nodes of each segment. The heat source is a function of the fuel irradiation history and the pin location within the bundle and the bundle's location within the reactor. A nominal power profile is input and multiplying factors are used to define heat loads for a particular pin within the pin bundle. In addition an axial distribution of power is also imposed on the fuel pin. A transient power profile is created using a time dependent multiplying factor that is input in tabular form. A sample profile corresponding to a normal scram is shown in Fig. 2. The transient coolant flowrate is specified in a similar manner.

\section{Execution Characteristics}

Because the thermal time constant is only $0.02 \mathrm{~s}$ the time step for the transient solution must also be small. An implicit, forward-backward differencing scheme is used for the thermal network solution. Because the solution is implicit there is no upper bound on the time step, however, if the time step greatly exceeds the thermal time constant, errors in the resulting calculation may become significant. The small thermal time constant results from large thermal couplings combined with low heat capacity within the model. The modeling of liquid metal coolants flowing at high Reynolds numbers around small diameter fuel pins with large internal heat generation leads to numerical instabilities in the solution. Especially significant are changes 


\section{NORMAL SCRAM}

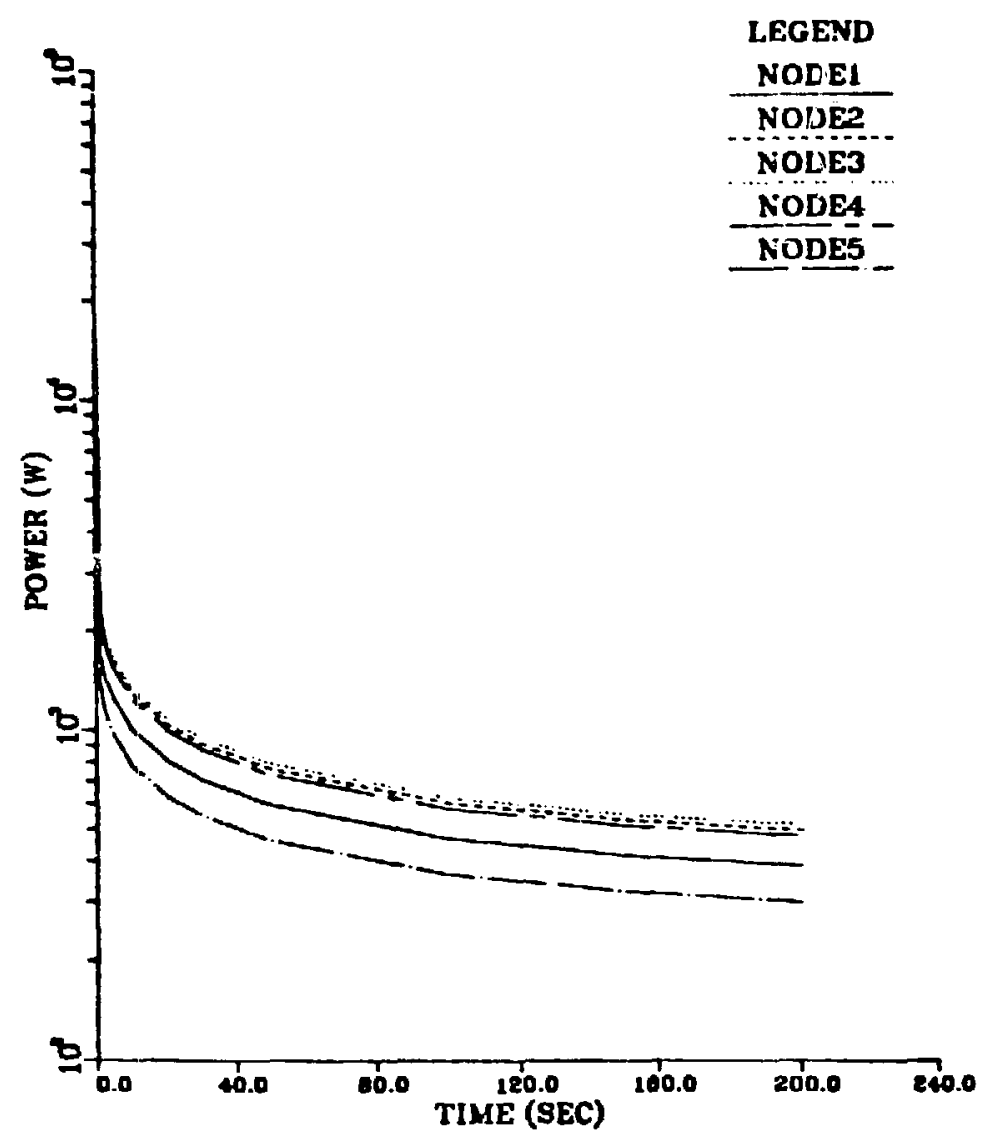

Fig. 2. Power profile for normal scram.

in the configuration that result in significant changes in the thermal couplings. Since the thermal conductance of the fuel and fuel-clad gap is strongly temperature dependent, numerical damping of these parameters must be utilized or severe temperature oscillations will occur. Because the use of these damping factors is largely empirical, sensitivity studies to determine their effect on the final results were conducted. In all cases the extent of the required damping had little influence on the final results.

This brief description of the model is intended to serve as an introduction to TRANSPA. Some of its special features and some of the difficulties encountered during its development were outlined. The following section contains a description of the output. The Appendix contains a listing of the program. 
TABLE I

TWELVE TRANSIENT EVENTS

Event

1. Normal scram

2. Rod drop

3. $0.5 \% / \mathrm{s}, 100 \%$ flow to $460 \mathrm{MW}$, primary trip

4. $3.4 \mathrm{~s} / \mathrm{s}, 100 \%$ flow to $486 \mathrm{MW}$, primary trip

5. Continuous flow reduction, primary trip

6. $0.5 \notin / \mathrm{s}, 100 \%$ flow to $500 \mathrm{MW}$, secondary trip

7. $3.4 \notin / \mathrm{s}, 100 \%$ flow to $526 \mathrm{MW}$, secondary trip

8. $25 \AA / s, 100 \%$ flow to $500 \mathrm{MW}$, primary trip

9. $25 \notin / \mathrm{s}, 100 \%$ flow to $528 \mathrm{MW}$, secondary trip

10. Total loss electric power

11. Continuous flow reduction, secondary trip

12. Primary pump seizure
Initial Condition

Ful1 power

Ful1 power

Full power

Full power

93\% power and flow

Ful 1 power

Fu11 power

Ful1 power

Full power

Ful1 power

93\% power and flow

Ful1 power

\section{OUTPUT AND RESULTS}

TRANSPA is used to evaluate the transient thermal response of a single fuel pin to the twelve events shown in Table I. For each event a transient power profile and coolant flowrate are specified. For each case a steady state solution is obtained that serves as the initial condition for the transient. The model then calculates the transient response of the fuel centerline, the outside clad, and the fluid outlet temperatures. These temperatures are saved on a separate file and plotted using a separate program. Examples of the output are shown in Figs. 3, 4, and 5. Each figure contains five curves corresponding to each of the five axial nodes in TRANSPA.

The model is set up to analyze all of the twelve transient events in a single run. Logic to accomplish this is contained in the MITAS execution 
NORMAL SCRAM

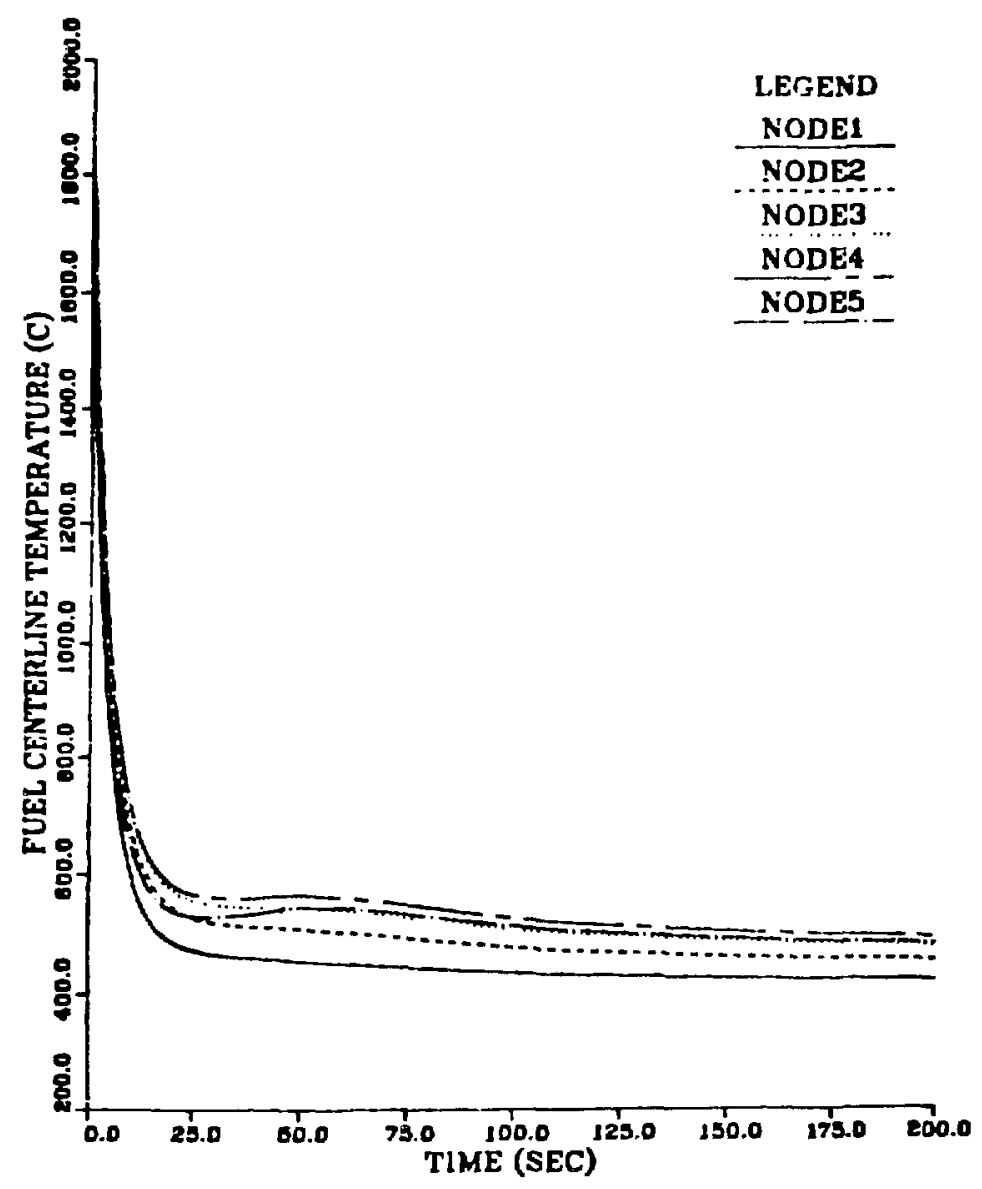

Fig. 3. Fuel centerline temperature for normal scram.
NORMAL SCRAM

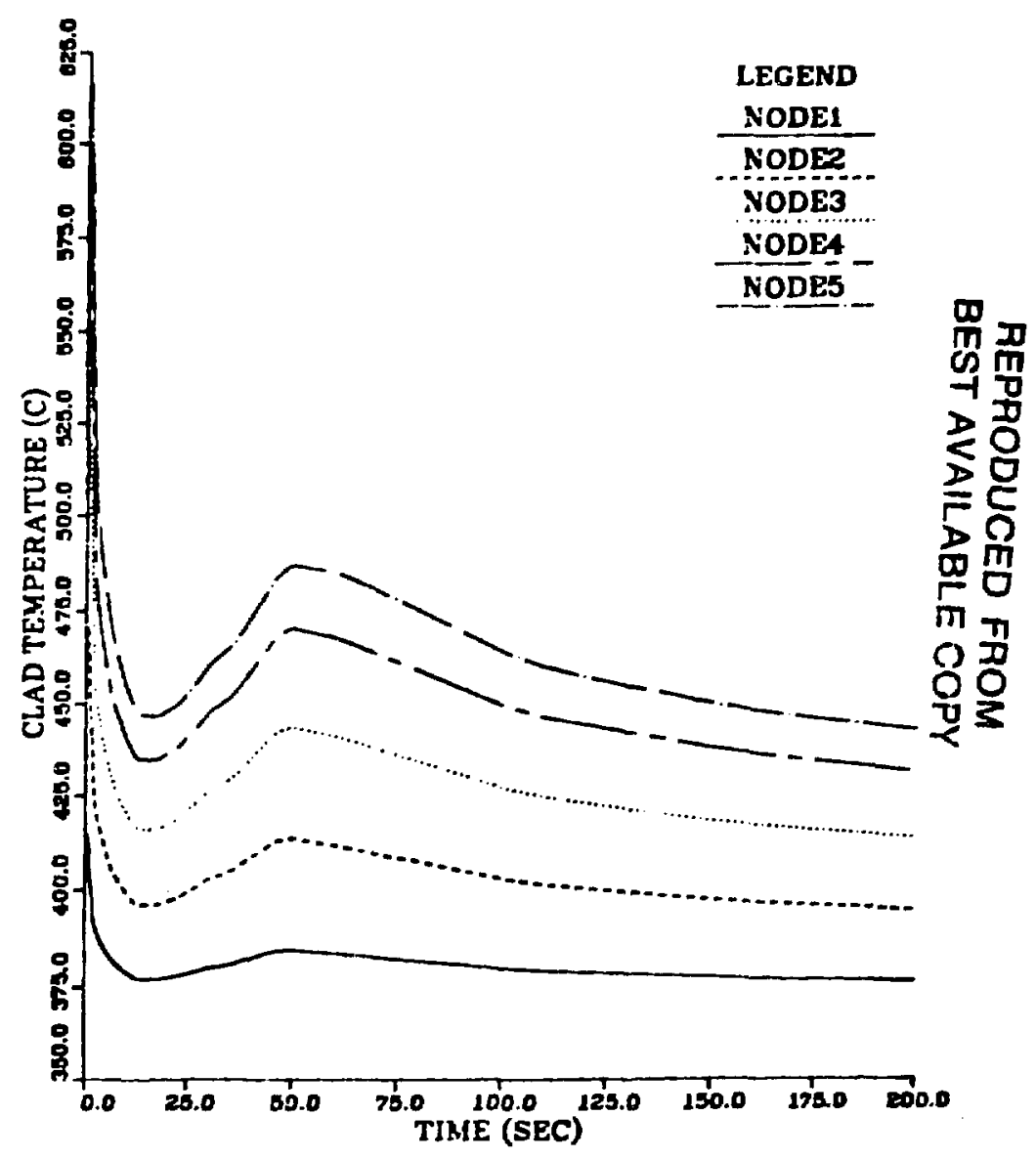

Fig. 4. Clad temperature for normal scram. 
NORMAL SCRAM

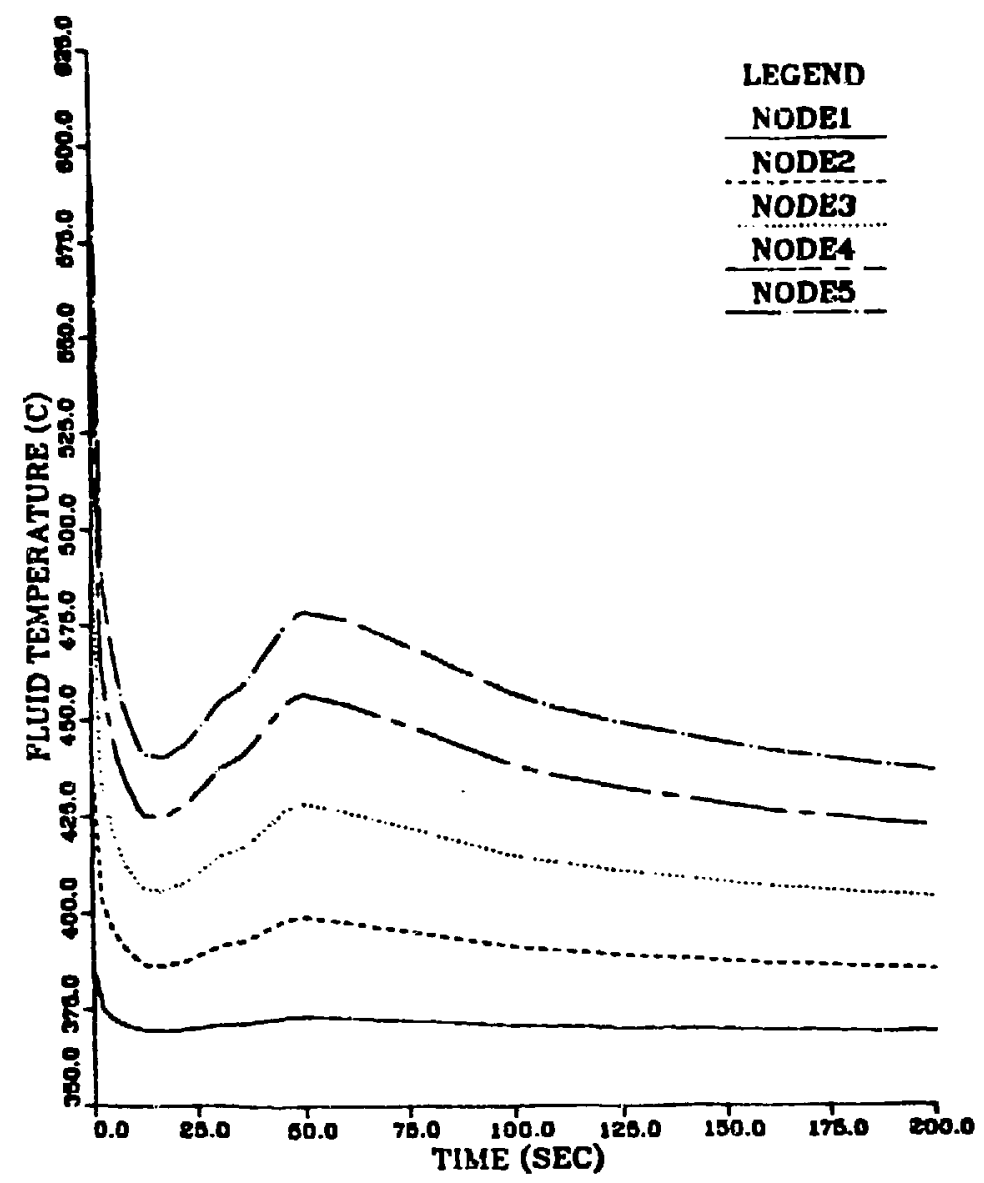

Fig. 5. Cool ant temperature for normal scram.

routine. In addition two steady state at $93 \%$ power and coolant flow rate conditions are analyzed. The solution time step is varied during each calculation depending on the rate of change of the power profile or coolant flowrate. Execution time for the twelve transient cases requires approximately ten minutes of central processing time on the NOS system.

\section{REFERENCES}

1. G. M. Holmstead, R. E. Kannady, Jr., R. J. Connor, and E. Shirley, "Martin Marietta Interactive Thermal Analysis System, MITAS-II, "User's Manual, May 1976. 
2. ABAQUS Computer Program Manuals (Hibbitt, Karlsson \& Sorensen, Inc., Providence, Rhode Island, 198T), Vol. 1-4.

3. E. P. Coomes, E. V. Khan, T. C. George, R. L. Cheatham, and D. R. Rector, "User's Manual, COBRA-WC: A Version of COBRA for Single Phase and Single and Multiassembly Thermal-Hydraulic Transient Analysis," Pacific Northwest Laboratories report PNL-3259, 1979.

4. T. L. George and K. L. Peddicord, "SPECKLE III: A Computer Code for Calculating the Behavior of Sphere Pac Fuel Pins," Oregon State University Rept. OSU-EIR-64, December 1982. 


\section{APPENDIX}

MITAS II INPUT DATA FOR TRANSPA MODEL 
DATE B4/09/24.TIME 17.16.04. MARTIN MARIETTA THERMAL ANALYZER SYSTEM

START OF INPUT DECK PROCESSING

INPUT CARD COL. $=12345678 \quad 1234567822345678 \quad 3234567842345678523456786234567872345678$ B EDIT NO.

BCD 3TITLE DATA

BCD GTRANSPA: TRANSIENT SINGLE PIN ANALYSIS

BCD GCARBIDE FUELS PROGRAM

BCD 9LANL MAY 1934

BCD 95 AXIAL. NODES W/ AXIAL CONDUCTION

BCO 9AC3 CONFIGURATION. PIN 10 (MAX TEMP)

BCD 9THERMAL CONOUCTIVITY IS CURVEFIT FR SPECKLE POROSITY $=0.23$

BCD 9 INCLUDES GAP CONDUCTANCE MODEL

BCD 9UNITS ARE G-CM-S-DEG $C-W$

END

$\begin{array}{rr}\text { A. } & 1 \\ \text { A. } & 2 \\ \text { A. } & 3 \\ \text { A. } & 4 \\ \text { A. } & 5 \\ \text { A. } & 6 \\ \text { A. } & 7 \\ \text { A. } & 8 \\ \text { A. } & 9 \\ \text { A. } & 10\end{array}$


DATE 84/09/24.TIME 17.16.04. MARTIN MARIETTA THERMAL ANAIYZER SYSTEM

TRANSPA: TRANSIENT SINGLE PIN ANALYSIS

CARBIDE FUELS PRQgRAM

LANL MAY 198

INPUT GARO COL. $=12345678 \quad 12345678223456783234567842345678523456786234567872345678$ 8 EDIT NO.

CARO NO.

BCD 3NODE DATA

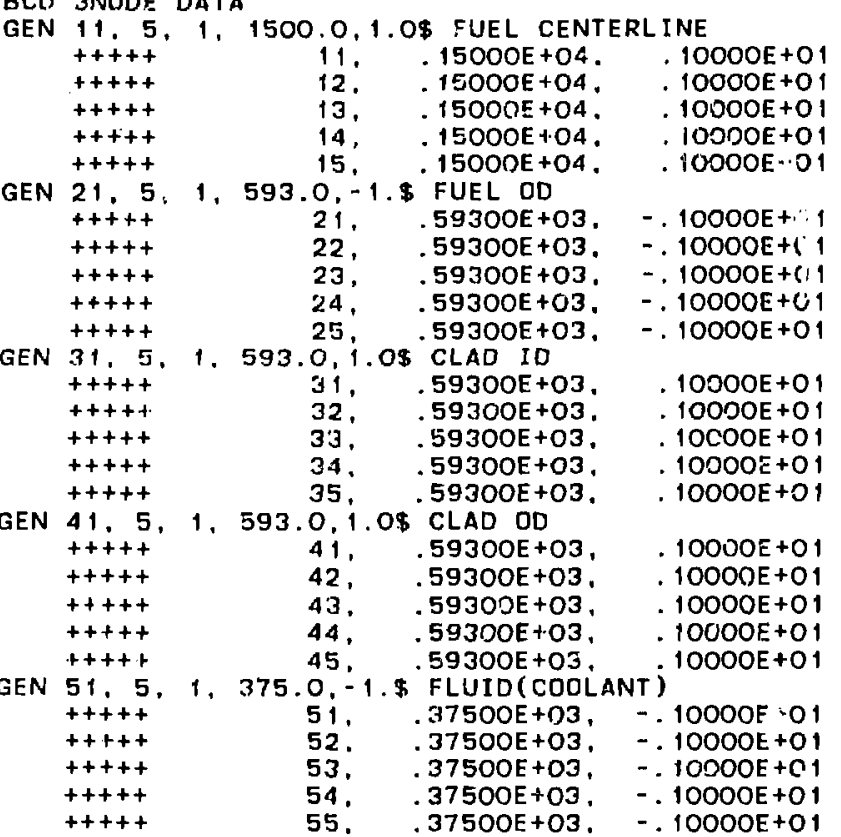

A. 11

RFL TO $116127 \mathrm{~B}$ REQUESTED. OLD FL=200000B. NEW FL=200000B. NODE SUMMARY

RFL TO $116127 \mathrm{~B}$ REQUESTED. OLD FL=200000B. NEW FL=200000B. NODE SUMMARY

THERE ARE 15 DIFFUSION NODES, 10 ARITHMETIC NODES, O HEATER NODES, AND 1 BOUNDARY NODES
FOR A TOTAL OF 26 NDDES IN THE NETWORK

NODE DIRECTORY

INPUT / INTERNAL

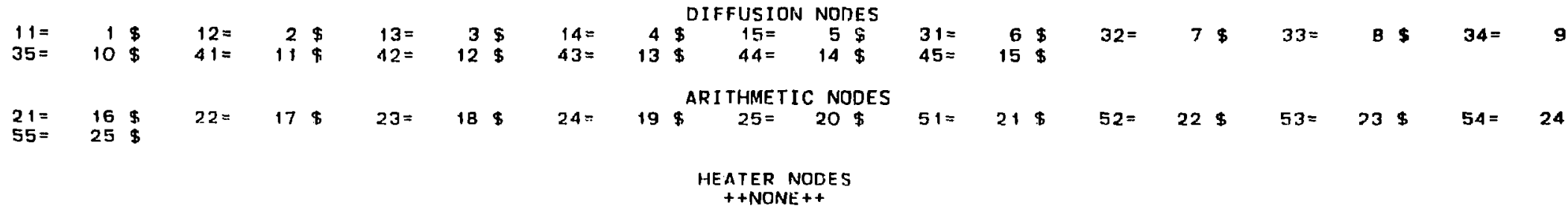


BCD 3CONDUCTOR DATA

RFL TO OOOOOOB REQUESTED. OLD FL $=200000 B$. NEW FL $=200000 B$

GEN $41,5,1,-50,4,41,1,0$ FLUID COUPLING

A. 19

A. 20

$\begin{array}{lrc}+++++ & 41, & -50 \\ +++++ & 42, & -51 \\ +++++ & 43, & -52 \\ +++++ & 44, & -53 \\ +++++ & 45, & -54 \\ 51,5,1,-50,1,51, & 1,0 \\ +++++ & 51, & -50 \\ +++++ & 52, & -51 \\ ++++ & 53, & -52 \\ +++++ & 54, & -53 \\ +++++ & 55, & -54\end{array}$

1. $.10000 E+01$

42. . 10000E+01

43. $10000+01$

45. $\quad .10000$ + +01

51. . 10000E+01

52. .10000E+01

53. 10000 E+O1

$55.10000+01$

GEN $61,5,1,-41,1,51,1,1.0$

$\begin{array}{lllll}+++++ & 61, & -41, & 51, & .10000 E+01 \\ +++++ & 62, & -42, & 52, & 10000 E+01\end{array}$

62, $+4++42, \quad 52, \quad .10000 E+01$

$+++++\quad 63, \quad-43, \quad 53, \quad .10000 \mathrm{E}+0$

t++t+ 64, $-44,54, \quad 6000 \mathrm{E}+0$

t+++t 65, $-45, \quad 55, \quad 10000 \mathrm{E}+0$

GEN $11,5,1,11,1,21,1,1.0 \$$ RADIAL CONDUCTION

$+++++11, \quad 11, \quad 21, \quad 10000 E+01$

$12, \quad 12, \quad 22, \quad .10000 E+0$

$13, \quad 13, \quad 23, .10000 E+01$

14, $14, \quad 24, .10000 E+0$

GEN $21,5,1,21,1,31,1,1,0^{15,} 25, \quad .10000 E+O$

t+++t $21, \quad 21, \quad 31, \quad .10000 \mathrm{E}+0$

$+++++22, \quad 22, \quad 32, \quad .10000 \mathrm{E}+0$

t+t+t 23, 23, 33, .10000E+0

$+++++\quad 24, \quad 24, \quad 34, \quad .10000 E+01$

t+t+t 25, 25; $35, \quad$. $10000 \mathrm{E}+0$

GEN $31,5,1,31,1,41,1,1.0$

$++++\quad 31, \quad 31 . \quad 41, \quad .10000 E+01$

$++++\quad 32, \quad 32, \quad 42, \quad .10000 E+01$

$++++\quad 33, \quad 33, \quad 43, \quad .10000 E+01$

it+t+ $34, \quad 34, \quad 44, \quad .100 D O E+01$

GEN1 $11,4,1,11,1,12,1,1.0 \$$ AXIAL CONDUCT ION

CONDUCTION

A. 21

A. 22

A. 23

A. 24

A. 25

A. 26

$\begin{array}{lllll}+++++ & 112, & 12, & 13, & .10000 E+01 \\ +++++ & 113, & 13, & 14, & .10000 E+01\end{array}$

$\begin{array}{lllll}+++++ & 113, & 13, & 14, & .10000 E+01 \\ +++t+ & 114, & 14, & 15, & 10000 E+01\end{array}$

GEN131,4,1.31,1.32.1:1.0

$++++\quad 31, \quad 32, \quad 31,10000 \mathrm{E}+01$

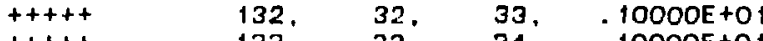

$+++++\quad 33, \quad 34, \quad .10000 E+0$

GEN141, 4.1,41,1,42, $134,34, \quad 35, .10000 E+0$

$\begin{array}{rrrr}++++ & 14, \\ +++++ & 49, & 42, & 10000+01\end{array}$
A.

A. 27

A. 28
20

21

22

23

24

26

9

1

23

25

26

27

28 
FFL TO $116135 B$ REQUESTED. OLD $F L=200000 B$. NEW $F L=200000 B$

CONDUCTOR SUMMARY

IMERE ARE 42 LINEAR CONDUCTORS AND O NDN-LINEAR CONDUCTORS, FOR A TOTAL DF 42 CONDUCTORS IN THIS NETWORK

\section{CONOUCTOR DIRECTORY}

INPUT = INTERNAL

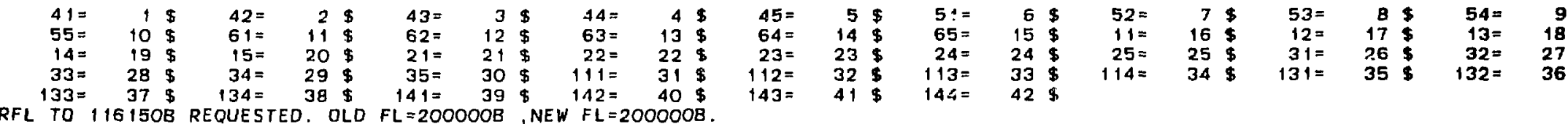

$\begin{array}{llllll}\text { RFL TO } & 116150 B & \text { REQUESTED. OLD } & F L=200000 B & \text { NEW } & F L=200000 B \\ \text { RFL TO } 1160438 & \text { REQUESTED. OLD } & F L=200000 B & \text { NEW } & F L=200000 B\end{array}$ 
INFUT CARO COL.12345678 $12345678 \quad 2 \quad 2345678 \quad 3 \quad 234567842345678 \quad 5 \quad 2345678 \quad 6 \quad 234567872345678$ 8 EDIT ND.

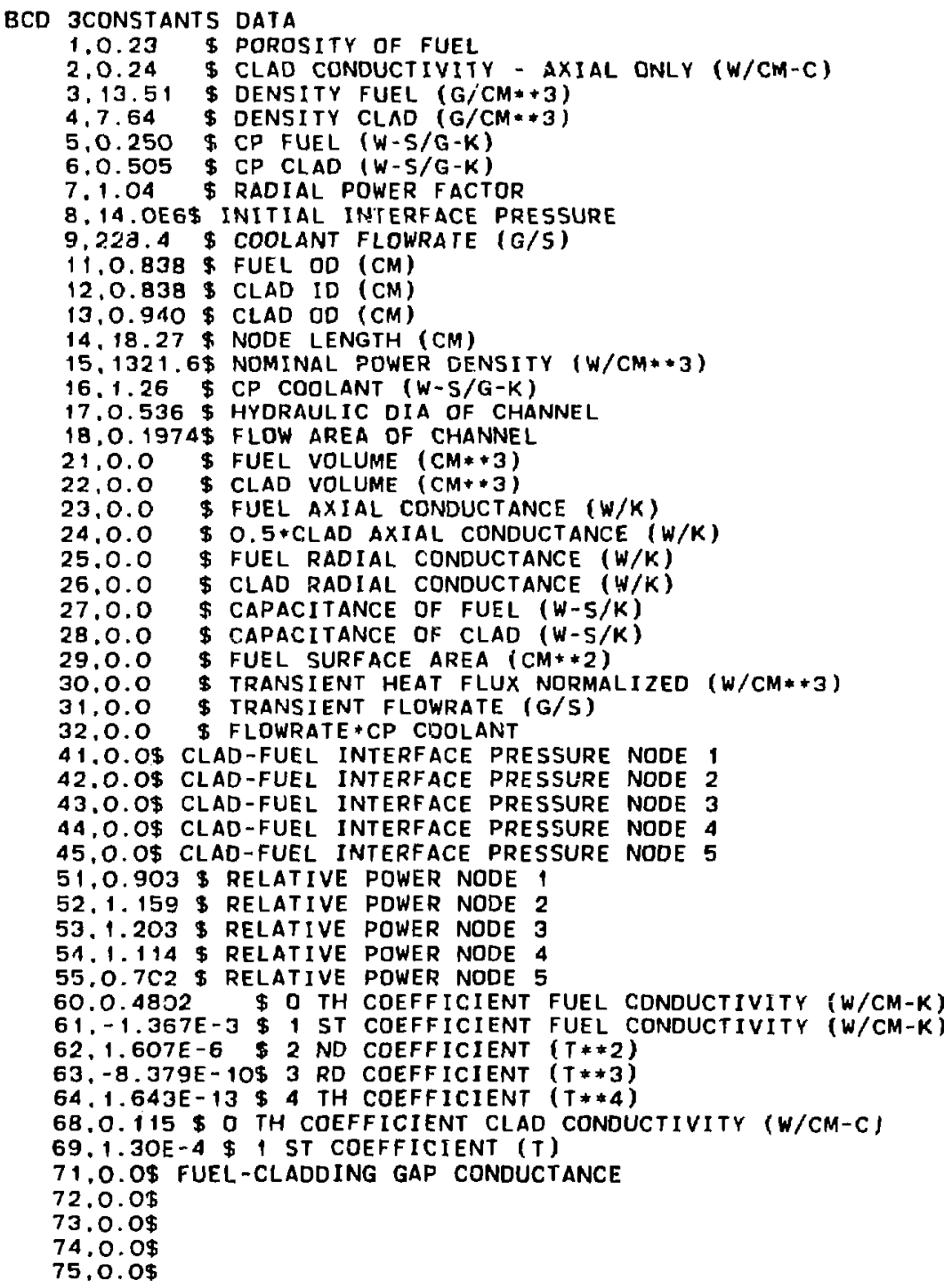




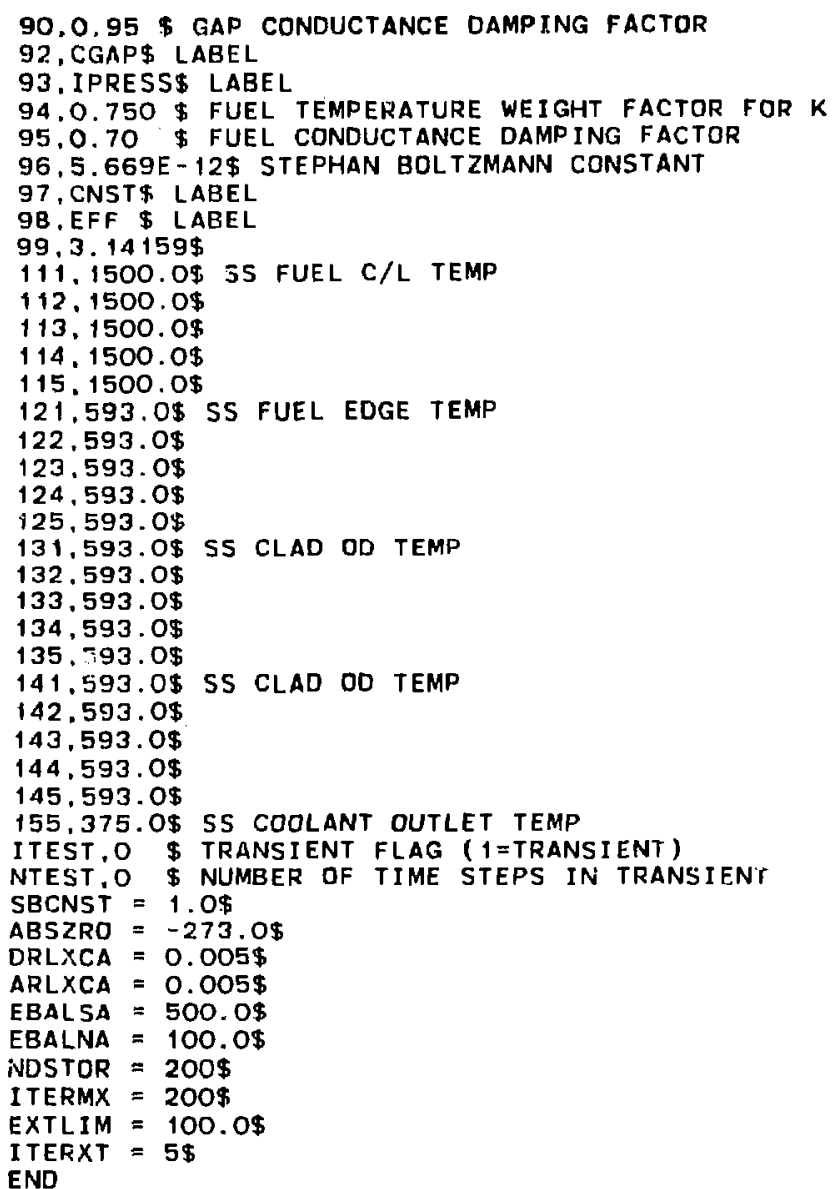

RFL TI) $116374 B$ REQUESTED. OLD FL=200000B. NEW FL $=200000 B$.

CONSTANTS DIRECTORY

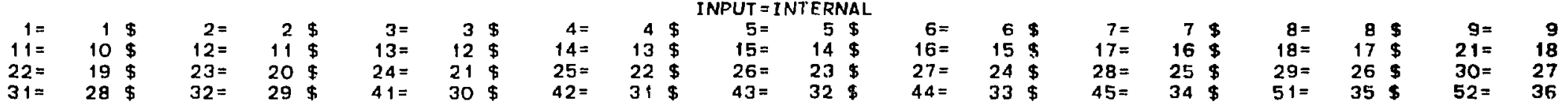




$60=40 \$ 61=41 \$ 62=$

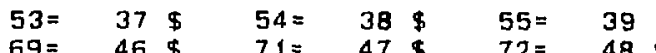

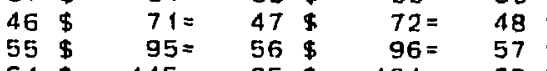

$14=64 \$ \quad 115=65 \$ \quad 121=66$

$133=73 \$ 134=74 \$ 135=75$

$61=41$

$98=59 \$$

$123=68$
$42 \$ 63=43$

$\begin{array}{rrr}51 \$ & 90= & 52 \\ 60 \$ & 111= & 61\end{array}$

$99=60$

$124=69$

$92=53993=54$

$\begin{array}{llll}155=70 \$ & 112= & 62 \$ & 113=6\end{array}$

$144=79 \quad 145=80 \$ 155=81$ 


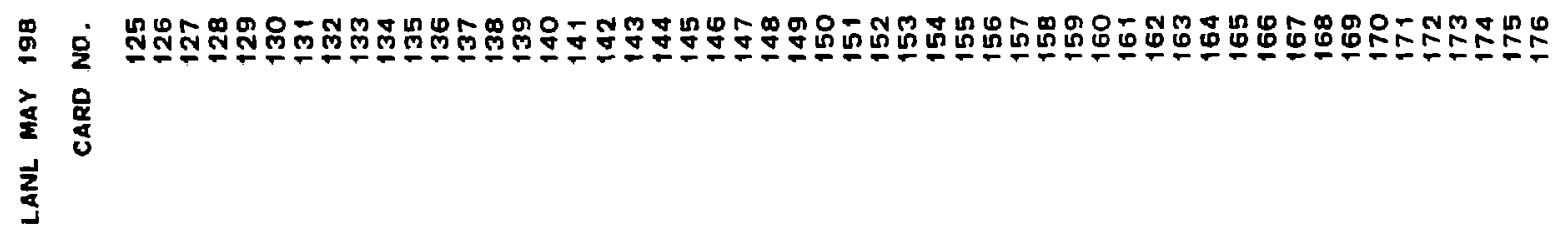

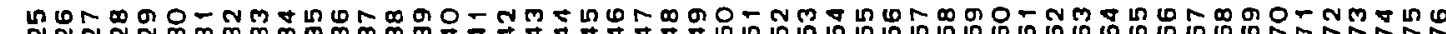

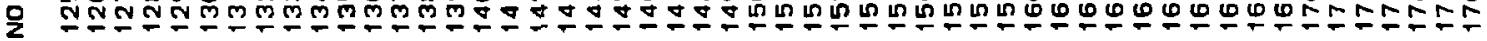

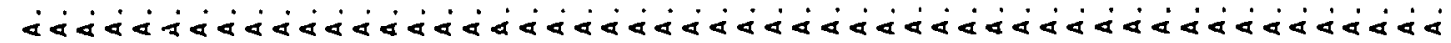

$\infty$

$\stackrel{\infty}{0}$

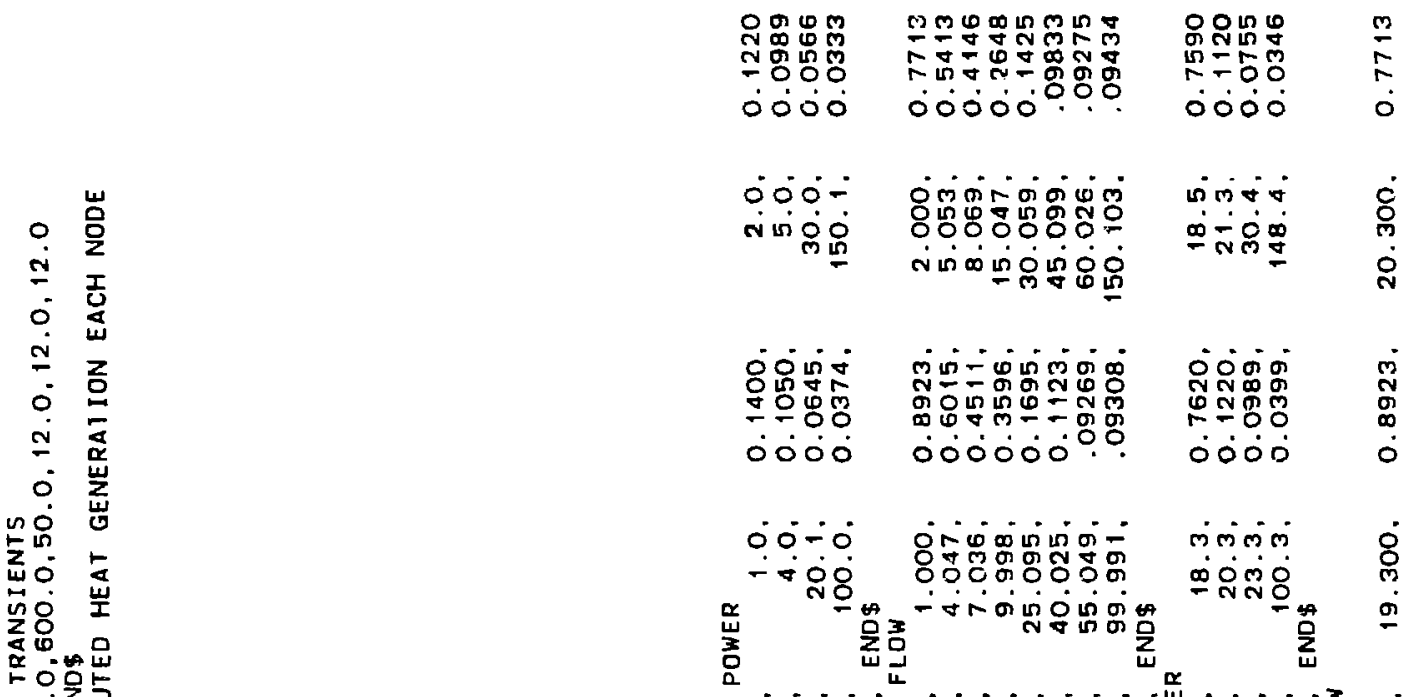

प्र.

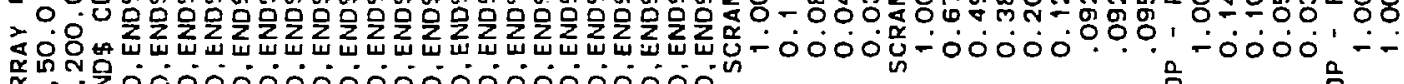

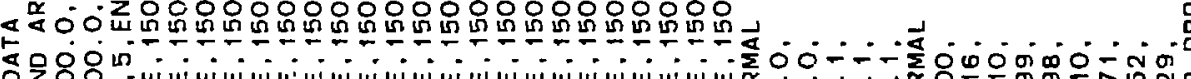




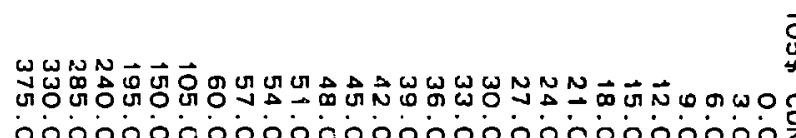

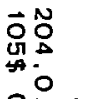

응

$\vec{\omega}_{N}$

WO

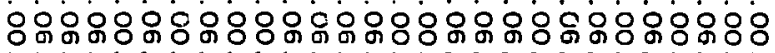

Z0

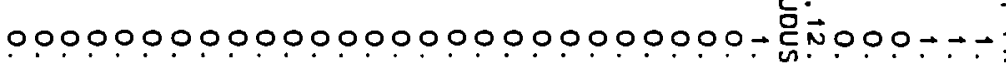

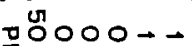

D. 000000

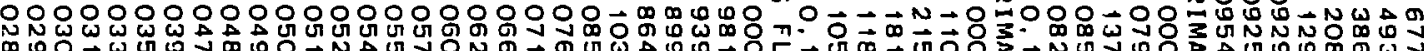

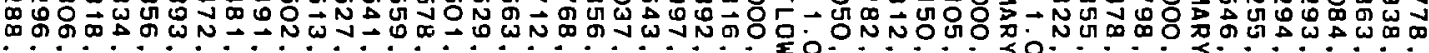

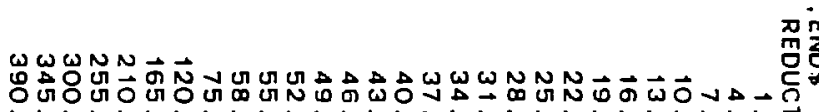

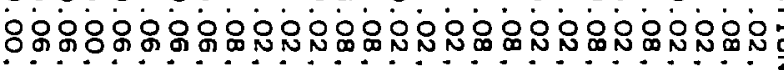

D

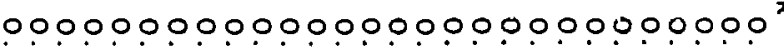

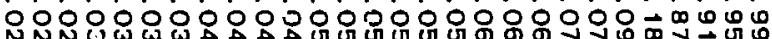

N N

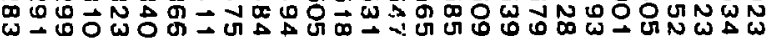

\section{중}

$\vec{n} \infty=0$

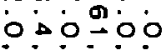

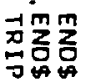

$=000$

००००

要金二

DZZUUANNN

in के के is

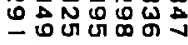

$0.000 \rightarrow$

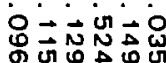

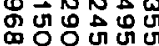

\section{$00 \rightarrow-$ \\ 용요 $=$ \\ ㄲํㅇ융ํํㅇ}

落

जัง

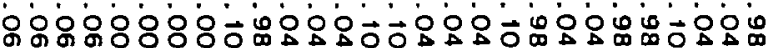

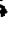
ดง UN

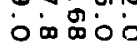
००००

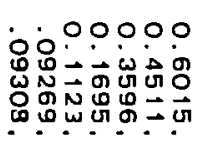
$\vec{T}$ O月品N .

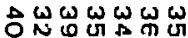
$\omega 0.040 \omega$

$0,00 \rightarrow+4$ $=\vec{N} \omega \overrightarrow{0}$

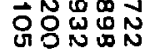

우 $\rightarrow$

웅응우

- 00000

000 in in D NAOA

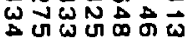
N

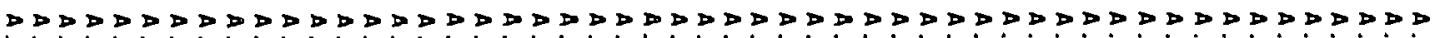




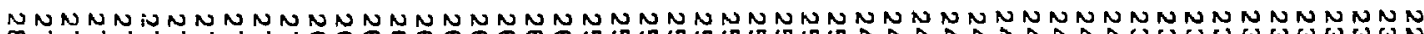

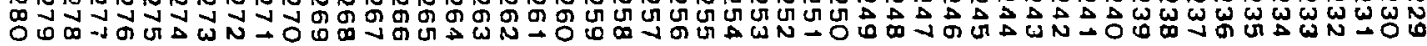




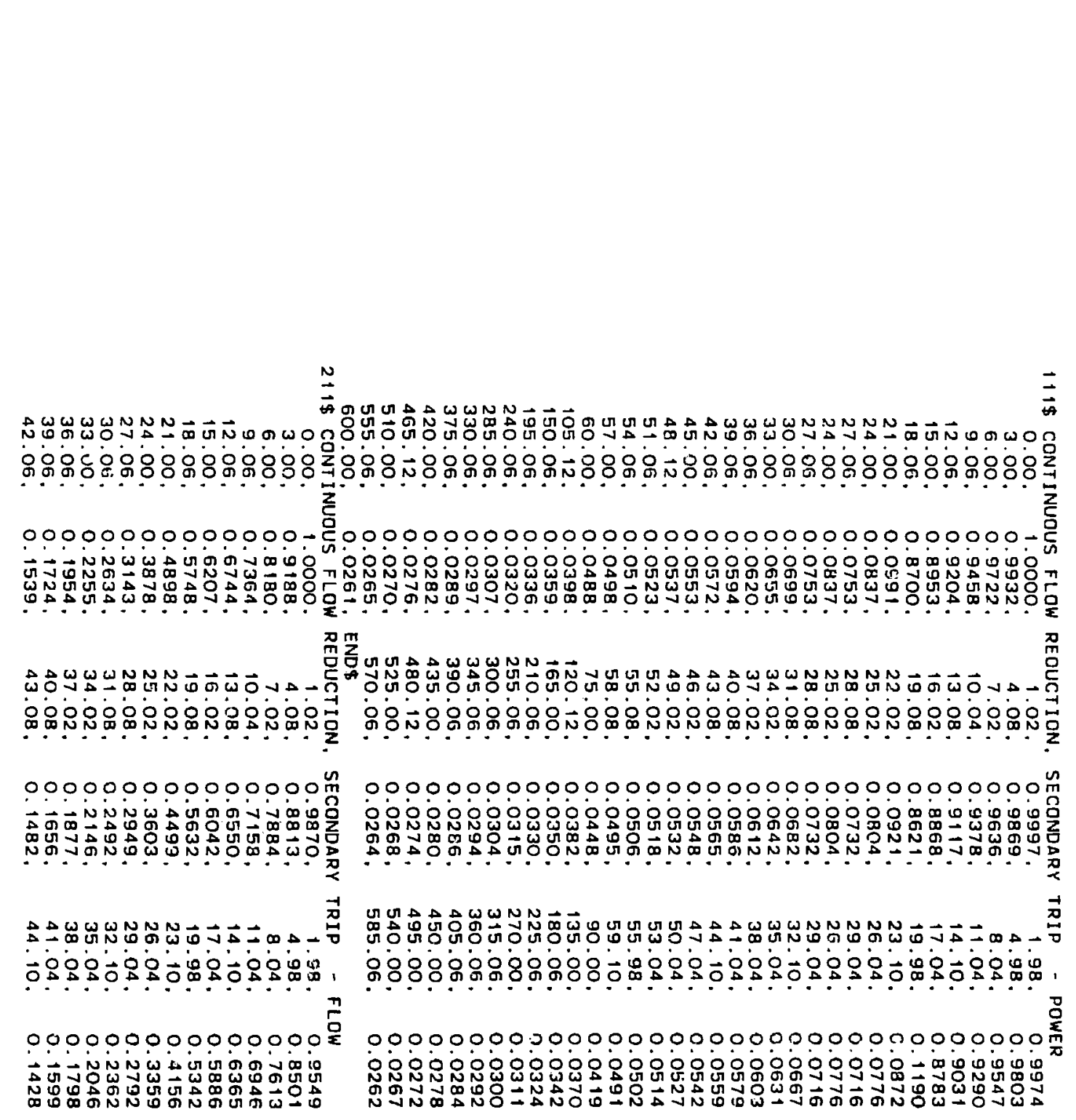

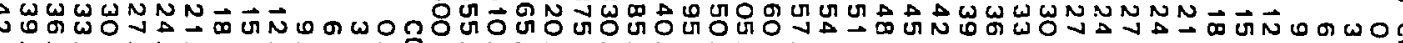

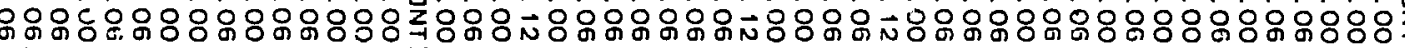
$00000000000000-\mathrm{C}_{\mathrm{L}} 0000000000000000000000000000000000+\mathrm{L}_{\mathrm{n}}$

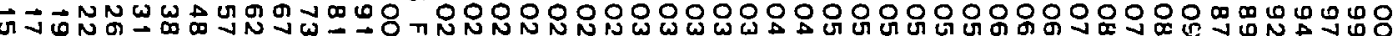
$\omega N$ N

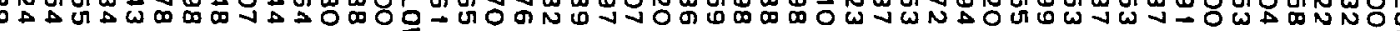

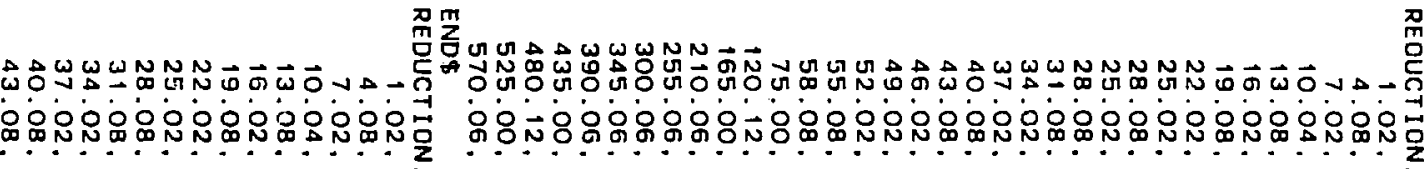
000000000000000 m $\vec{A} \vec{N} N$

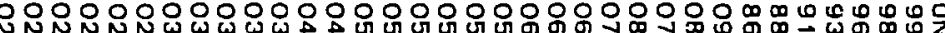
西

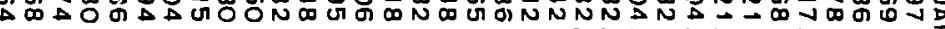

Un Un $A \rightarrow \omega \omega N N \vec{A}-1$ Noún

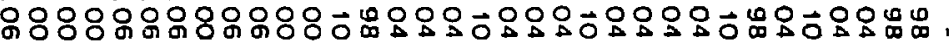

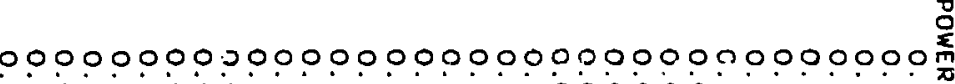

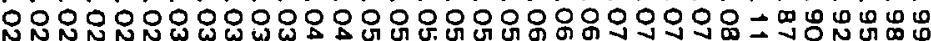

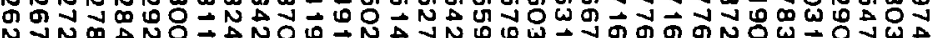

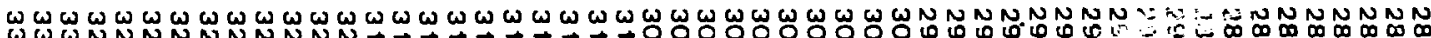

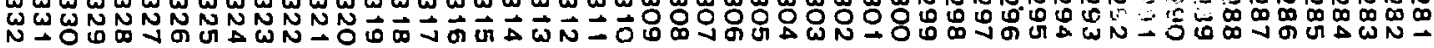




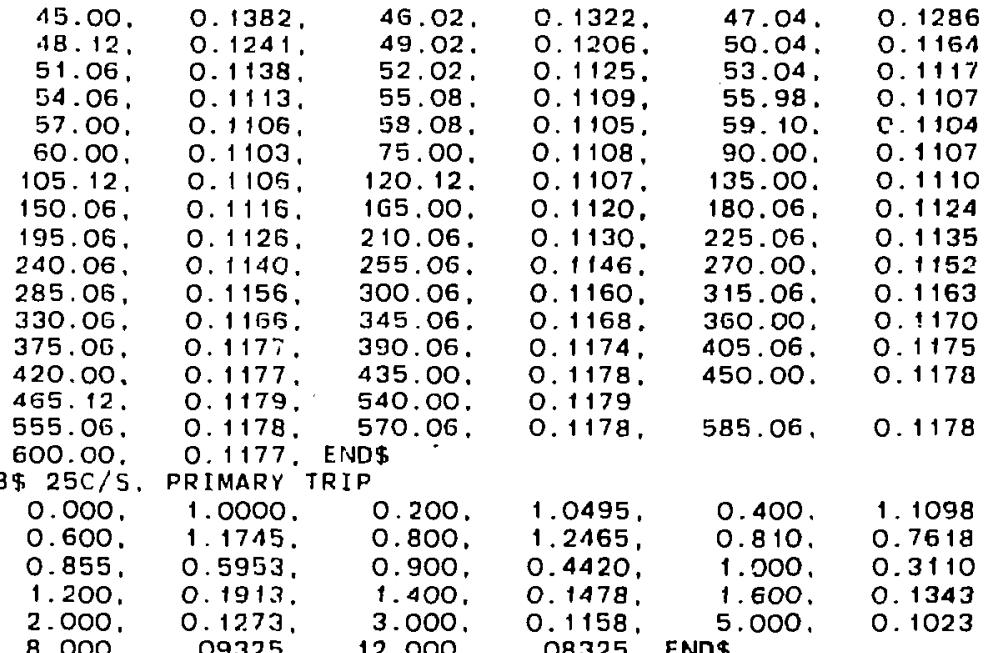

$8.000,09325,12.000$.

$208,0.0,1,0,12,0,1,0$, END\$

$209,0.0,1,0,12,0,1,0$, ENDS

110 T TOTAL LOSS ELECTRIC POWER, - POWER

\begin{tabular}{|c|c|c|c|c|c|}
\hline $\begin{array}{l}0.0 . \\
3.0 \\
0.1\end{array}$ & $\begin{array}{l}1.0000, \\
0.1120, \\
0.0800, \\
0.0474\end{array}$ & $\begin{array}{r}1.0 \\
4.0 \\
20.1\end{array}$ & $\begin{array}{l}0.1400, \\
0.1050, \\
0.0645\end{array}$ & $\begin{array}{r}2.0 \\
5.0 \\
30.0\end{array}$ & \\
\hline
\end{tabular}

50.1, $0.0474, \quad 100.0,0.0374$

$210 \$$ TOTAL LOSS ELECTRIC POWER, - FLOW

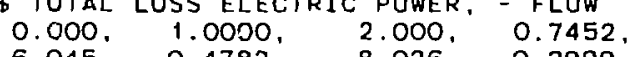

$6.045, \quad 0.4782, \quad 8.026, \quad 0.3999$,

14.081. $0.2550, \quad 20.152, \quad 0.1809$.

4.093, $\quad 0.5813$

$10.119, \quad 0.3370$

30.143, 0.1166, 40.144, .08051, 50.158, .05880

$60.061, \quad .04532,70.185, \quad .03564,78.128, \quad .02961$

$90.075, .02743,100.021, .02741,150.134, .02966$

$112 \$$ PRIMARY PUMP SEIZURE, - POWER

$\begin{array}{llllll}0.000, & 1.0000, & 1.000 . & 0.2758, & 2.000 . & 0.1216 \\ 3.001, & 0.1114 & & & & \\ 4.001 . & 0.1042 . & 5.014 . & .09829, & 6.041 . & .09328 \\ 7.061 . & .08910 . & 8.025 . & .08571 . & 9.025 . & .08267\end{array}$

A. $\quad 333$ 
INPUT CARD COL. $=12345678$ I 23456782234567832345678 \& 2345678523456786234567872345678 B ED1T NO.

CARD NO.

\begin{tabular}{|c|c|c|c|c|c|}
\hline $\begin{array}{l}\text { 10.058, } \\
25.134 \\
40.001 \\
150.077 \\
25 \text { PR IMARY }\end{array}$ & $\begin{array}{l}.07995 \\
.05998 \\
.05129 \\
.03328 \\
\text { PUMP SE }\end{array}$ & $\begin{array}{l}15.095 \\
29.978 \\
50.123 \\
200.128\end{array}$ & $\begin{array}{l}.07049 \\
.05659 \\
.04738 \\
.03095, \mathrm{E}\end{array}$ & $\begin{array}{l}20.027 \\
35.006 \\
100.073 \\
10 \$\end{array}$ & $\begin{array}{r}.06452 \\
.05370 \\
.03742\end{array}$ \\
\hline 0.000 & 1.0000 & 1.000 & 0.8244 & 2.000 & 0.7490 \\
\hline $\begin{array}{r}4.001 \\
7.061 \\
10.058 \\
25.134 \\
40.001 \\
150.077\end{array}$ & $\begin{array}{l}0.5766 . \\
0.4230 \\
0.3336 \\
0.1551 \\
.09723 \\
.08312\end{array}$ & $\begin{array}{r}5.014 \\
8.025 \\
15.095 \\
29.978 \\
50.123 \\
200.128\end{array}$ & $\begin{array}{l}0.5153 \\
0.3897 \\
0.2437 \\
0.1307 \\
.08238 \\
.08410\end{array}$ & $\begin{array}{r}6.041 \\
9.025 \\
20.027 \\
35.006 \\
100.073 \\
\text { ND\$ }\end{array}$ & $\begin{array}{l}0.4647 \\
0.3600 \\
0.1911 \\
0.1118 \\
.08203\end{array}$ \\
\hline
\end{tabular}

END

$150.077^{\circ}, 08312, \quad 200.128, .08410$, END\$ 2.08203

RFL TO $116231 \mathrm{~B}$ REQUESTED, DLD FL $=200000 B$.NEW FL $=200000 B$.

$\begin{array}{ll}\text { A. } & \mathbf{3 8 5} \\ \text { A. } & \mathbf{3 8 6} \\ \text { A. } & \mathbf{3 8 7} \\ \text { A. } & \mathbf{3 8 8} \\ \text { A. } & \mathbf{3 8 9} \\ \text { A. } & 390 \\ \text { A. } & 391 \\ \text { A. } & 392 \\ \text { A. } & \mathbf{3 9 3} \\ \text { A. } & \mathbf{3 9 4} \\ \text { A. } & \mathbf{3 9 5} \\ \text { A. } & \mathbf{3 9 6} \\ \text { A. } & \mathbf{3 9 7} \\ \text { A. } & \mathbf{3 9 8}\end{array}$

ARRAY OIRECTORY
INPUT NO.
STARTING LOCATION
INTERNAL NO.
NO. OF ELEMENTS

$\begin{array}{rr}2 & 1 \\ 8 & 14 \\ 40 & 20 \\ 41 & 171 \\ 42 & 322 \\ 43 & 473 \\ 44 & 624 \\ 45 & 775 \\ 51 & 926 \\ 52 & 1077 \\ 53 & 1228 \\ 54 & 1379 \\ 55 & 1530 \\ 61 & 1631 \\ 62 & 1832 \\ 63 & 1933 \\ 64 & 2134 \\ 65 & 2285 \\ 66 & 2436 \\ 71 & 2587 \\ 72 & 2738 \\ 73 & 2889 \\ 74 & 3040 \\ 75 & 3191 \\ 101 & 3342 \\ 201 & 3369 \\ 102 & 3420 \\ 202 & 3447 \\ 103 & 3500 \\ 203 & 3527 \\ 104 & 3532 \\ 204 & 3567 \\ 105 & 3572\end{array}$

$\begin{array}{rr}1 & 13 \\ 2 & 6 \\ 3 & 151 \\ 4 & 151 \\ 5 & 151 \\ 6 & 151 \\ 7 & 151 \\ 8 & 151 \\ 9 & 151 \\ 10 & 151 \\ 11 & 151 \\ 12 & 151 \\ 13 & 151 \\ 14 & 151 \\ 15 & 151 \\ 16 & 151 \\ 17 & 151 \\ 18 & 151 \\ 19 & 151 \\ 20 & 151 \\ 21 & 151 \\ 22 & 151 \\ 23 & 151 \\ 24 & 151 \\ 25 & 27 \\ 26 & 51 \\ 27 & 27 \\ 28 & 53 \\ 29 & 27 \\ 30 & 5 \\ 31 & 35 \\ 32 & 5 \\ 33 & 195\end{array}$


$\underset{N}{N}$

TRANSP: TRANSIENT SINGLE PIN ANALYSIS

$\begin{array}{lll}205 & 3757 & 34 \\ 105 & 39.14 & 35 \\ 206 & 3971 & 36 \\ 107 & 3976 & 37 \\ 207 & 4011 & 38 \\ 111 & 4016 & 39 \\ 211 & 4223 & 40 \\ 108 & 4410 & 41 \\ 208 & 4445 & 42 \\ 109 & 4450 & 43 \\ 209 & 4483 & 44 \\ 110 & 4488 & 45 \\ 210 & 4515 & 46 \\ 112 & 4554 & 47 \\ 212 & 4597 & 48\end{array}$

CARBIDE FUELS PROGRAM

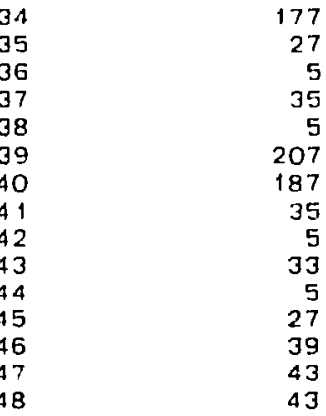

REL TO 1271308 REQUESTED. OLD FL =200000B. NEW FL $=200000 B$. 
RFL TO $116376 B$ REQUESTED, OLD FL $=200000 B$. NEW FL $=2000008$.

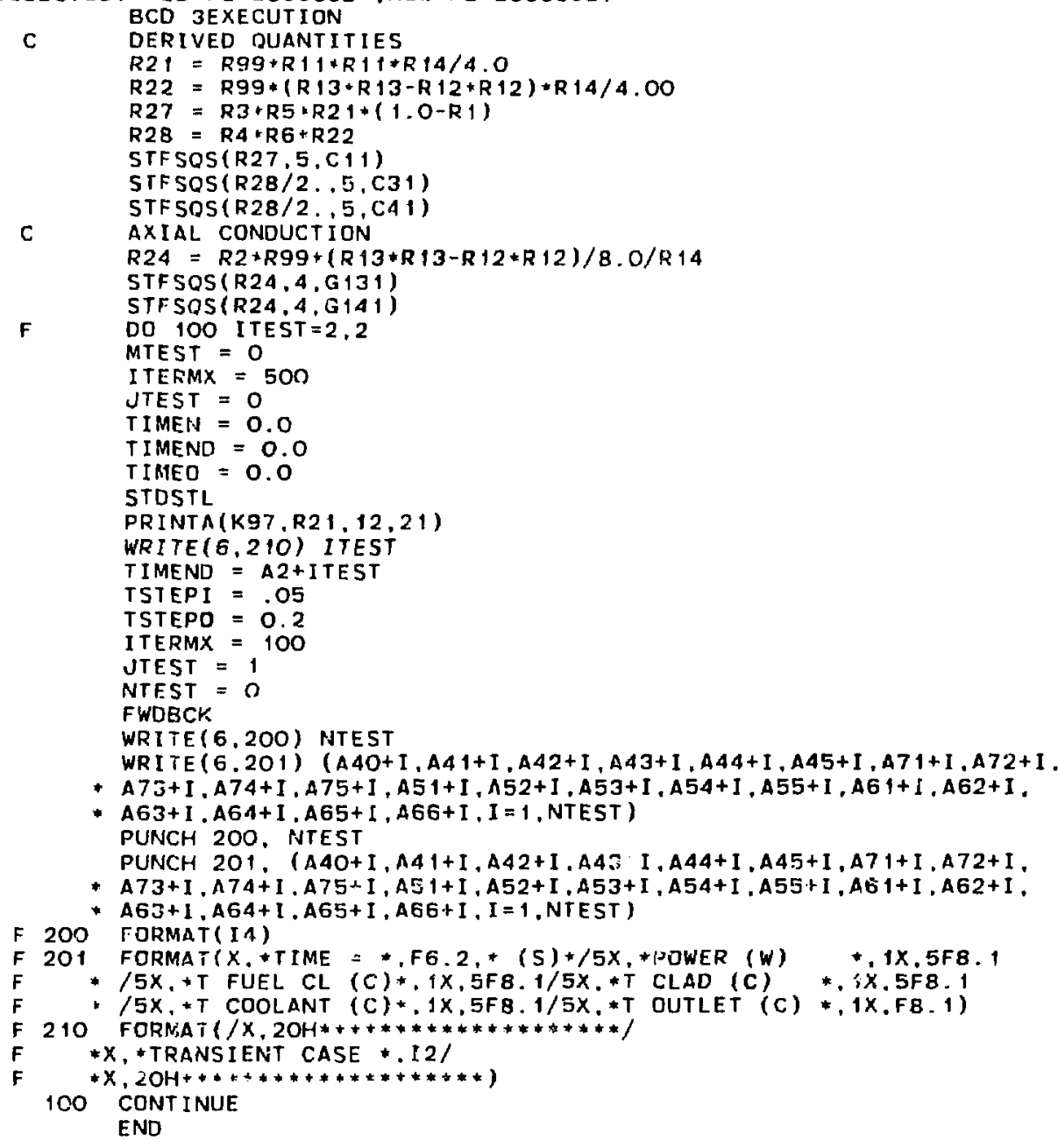

\begin{tabular}{|c|c|c|}
\hline A. & 399 & $\begin{array}{l}399 \\
400\end{array}$ \\
\hline A. & 400 & 401 \\
\hline A. & 401 & 402 \\
\hline A. & 402 & 403 \\
\hline A. & 403 & 404 \\
\hline A. & 404 & 405 \\
\hline A. & 405 & 406 \\
\hline A. & 406 & 407 \\
\hline A. & 407 & $40 B$ \\
\hline A. & 408 & 409 \\
\hline A. & 409 & 410 \\
\hline A. & 410 & 411 \\
\hline A. & 411 & 412 \\
\hline A. & $\begin{array}{l}412 \\
413\end{array}$ & 413 \\
\hline A. & $\begin{array}{l}413 \\
414\end{array}$ & 414 \\
\hline A. & 415 & 415 \\
\hline A. & 416 & 416 \\
\hline A. & 417 & $\begin{array}{r}417 \\
418\end{array}$ \\
\hline A. & 418 & $\begin{array}{r}478 \\
419\end{array}$ \\
\hline A. & 419 & 420 \\
\hline A. & 420 & 421 \\
\hline A. & 421 & 422 \\
\hline A. & 422 & 423 \\
\hline A. & 423 & 424 \\
\hline A. & 424 & 425 \\
\hline A. & 425 & 426 \\
\hline A. & 426 & 427 \\
\hline A. & 427 & 428 \\
\hline A. & 428 & 429 \\
\hline A. & 429 & 430 \\
\hline A. & 430 & 431 \\
\hline A. & 431 & 432 \\
\hline A. & 432 & 433 \\
\hline A. & 433 & 434 \\
\hline A. & 434 & 435 \\
\hline A. & 435 & 436 \\
\hline A. & 436 & 437 \\
\hline$\Delta$ & 437 & 438 \\
\hline A. & 438 & 439 \\
\hline A. & 439 & 440 \\
\hline A. & 440 & 441 \\
\hline A. & 441 & 442 \\
\hline A. & 442 & 443 \\
\hline A. & 44.3 & 444 \\
\hline A. & 444 & 445 \\
\hline A. & 445 & \\
\hline
\end{tabular}


BCD 3VARIABLES 1

IF(TIMEN. LE.5.O) GO TO 10

TSTEPI $=0.05$

TSTEPO $=0.5$

IF (TIMEN. LE . 10.0) GO TO 10

TSTEPO $=1.0$

IF (TIMEN. LE.50.0) GO TO 10

TSTEPI $=0.5$

TSTEPO $=10.0$

10 CONTINUE

CONDUCTION IIN\FUEL

TFUEL $1=R 94+T 11+(1.0-R 94)+T 21$

TFUEL $2=R 94+T 12+(1.0-R 94)+T 22$

TFUEL3 $=R 94+T 13+(1.0-R 94)+T 23$

TFUEL4 $=$ R94*T14+(1.0-R94) *T24

TFUEL5 $=$ R94 +T15 + (1.0-R94) +T25

CFUEL (TFUEL1, R14, R11,R1, R6O,R61,R62,R63, R64,GFUEL1,G111)

CFUEL (TFUEL2, R14 R11,R 1, RGO R61, R62,R63,RG4,GFUEL $2, G 112$ )

CFUEL(TFUEL3,R14,RIH,R R R60,R61,RG2,RG

CFUEL (TFUELA,R14,K 1 , RI,RGO,RG

, $1, R 1, R G 0, R G 1, R 62, R 63, R 64, G F U E L 4, G(13)$

CFUEL(TFUEL5, R 1A, R 11, R 1, R6O, RG 1, R62,R63, R64,GFUEL5,G 114 )

$D F=1.0$

IF (JTEST.EO.O) DF $=$ R95

$G 11=D F * G F U E L 1+(1.0-D F) * G+1$

$G 12=D F+G F U E L 2+(1.0-D F)+G 12$

G13 $=$ DF *GFUEL3 $+(1.0-D F) * G 13$

G14 $=$ DF*GFUEL4 $*(1.0-D F) * G 14$

G15 = DF + GFUEL $5+(1 . D-D F)+G 15$

C RADIAL CONDUCTION IN CLAD

$C L A D=2.0 * R 99 * R 14 / A L O G(R 13 / R 12)$

$G 31=(R 6 B+R G Q *(T 31+T 4 i) / 2, * C L A D$

$\mathrm{G} 32=(\mathrm{R} 68+\mathrm{R} 69 *(\mathrm{~T} 32+\mathrm{T} 42) / 2) * \mathrm{CLAD}$

$G 33=(R G B+R 69+(T 33+T 43) / 2) * C L A D$

G33 $=(R G B+R 69 *(T 33+T 43) / 2) * C L A$.
$G 34=(R 6 B+R 69+(T 34+T 44) / 2) * C L A$.

G34 $=(R 6 B+R 69 *(T 34+T 44) / 2) * C L A$.
G35 $=(R 68+R 69+(T 35+T 45) / 2) * C L A$.

FUEL/CLAD GAP CONDUCTANCE

IF (JIEST.GT.O) GO TO 30

$R 111=T 11$

$R 112=T 12$

$R 113=T 13$

$R 114=T 14$

$R 115=T 15$

$R 121=T 21$

$R 122=T 22$

$R 123=T 23$

$R+23=T 23$
$R+24=T 24$

$R+25=T 25$

$R 131=T 31$

$R 132=T 32$

$R 133=T 33$

$R 134=T 34$

$R 134=T 34$
$R 135=T 35$ 
INPUT CARD COL. $=1234567812345678223456783234567842345678523456786234567872345678$ E EIT NO.

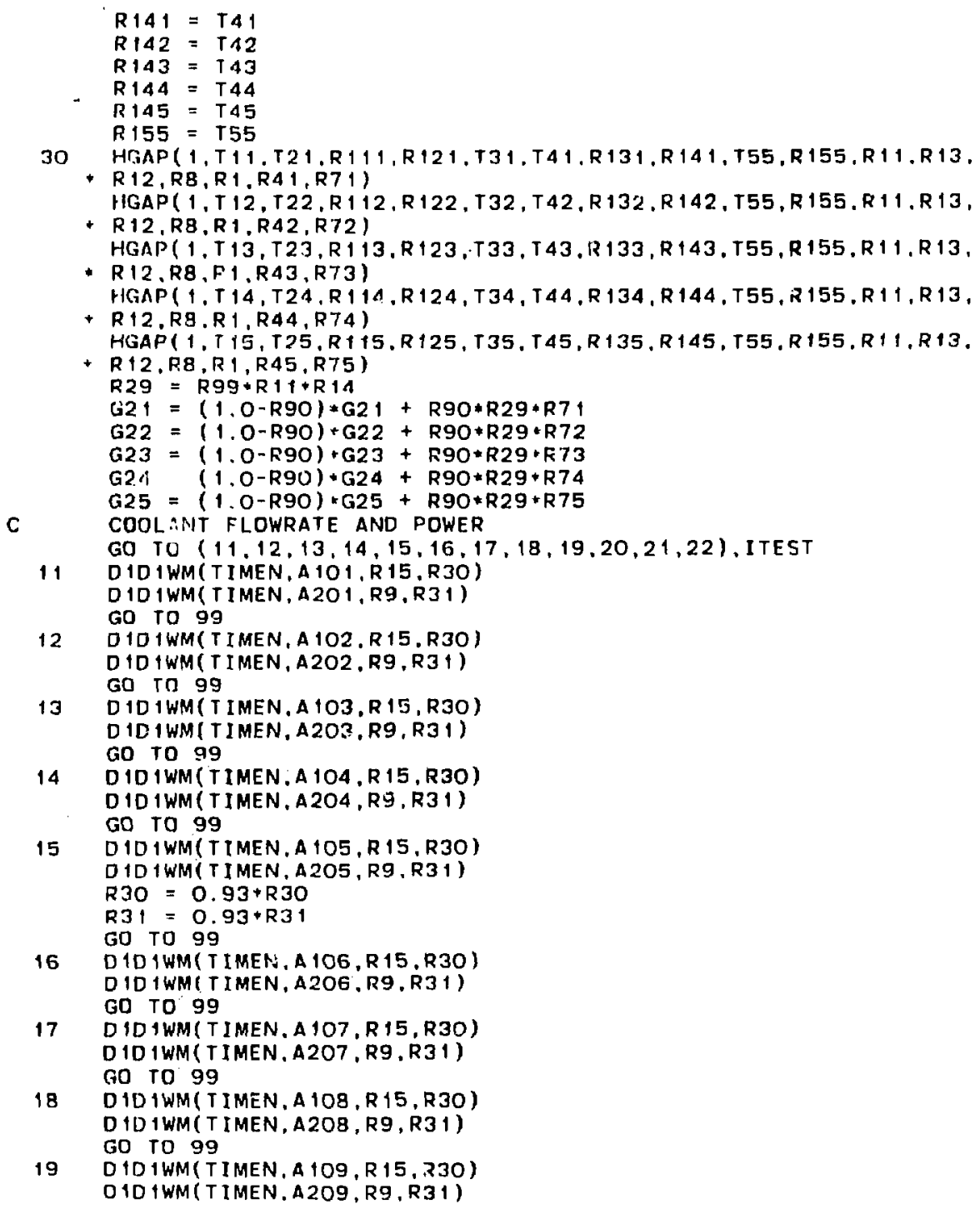

A. 498

A. 499

A. 500

A. 502

A. 503 
Go To 99

20 D1D 1WM( T IMEN, A 1 10, R 15, R30)

D 1D IWM (T IMEN, A2 10,R9,R3 i)

GO TO 99

A. 550

550

D 10 IWM(TIMEN, A 111, R 15, R3O)

D IDIWM(TIMEN, A2 $11, R 9, R 31)$

$R 30=0.93 * R 30$

$R 31=0.93 * R 31$

GO TO 99

22 DID1WM(TIMEN, A 1 12, R 15, R3O)

D 101WM(TIMEN, A2 12, R9, R31)

GO TO 99

99 CONT INUE

$R 32=R 31 * R 16$

HEAT GENERATION IN PIN

$011=R 7 * R 30+R 21 * R 51$

OII = R7*RJO*R21*R5

$012=$

$Q 13=R 7 * R 30 * R 21 * R 53$
$Q 14=R 7 * R 30 * R 21 * R 54$

$Q 15=R 7+R 30 * R 21 * R 55$

$A B+1=011$

$A B+2=012$

$A B+3=013$

$A B+4=014$

$A B+5=015$

C FLUID NETWORK

FILM (T50, T51,R32,R17, R18,R13,R14,G41, G61, G51)

FILM(T51,T52,R32,R17,R1B, R13,R 14, G42, G62, G52)

FILM(TS2, T53,R32,R17, R18,R13,R14,G43,G63,G53)

FILM(TS2, T53,R32,R $17, R 18, R 13, R 14, G 43, G 63, G 53)$

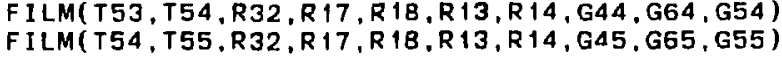

A. 551

A. $\quad 552$

A. $\quad 553$

A. $\quad 555$

A. $\quad 556$

A. $\quad 556$

A. $\quad 557$

A. 558

A. $\quad 559$

A. 561

A. $\quad 562$

A. 563

A. 564

A. 565

A. 566

A. $\quad \mathbf{5 6 6}$

A. $\quad 568$

A. 569

A. $\quad \mathbf{5 7 0}$

A. $\quad 571$

A. $\quad 572$

A. $\quad 573$

A. $\quad 574$

A. $\quad 575$

A. $\quad 576$

A. $\quad 577$

A. $\quad 578$

END

580

A. $\quad 581$ 


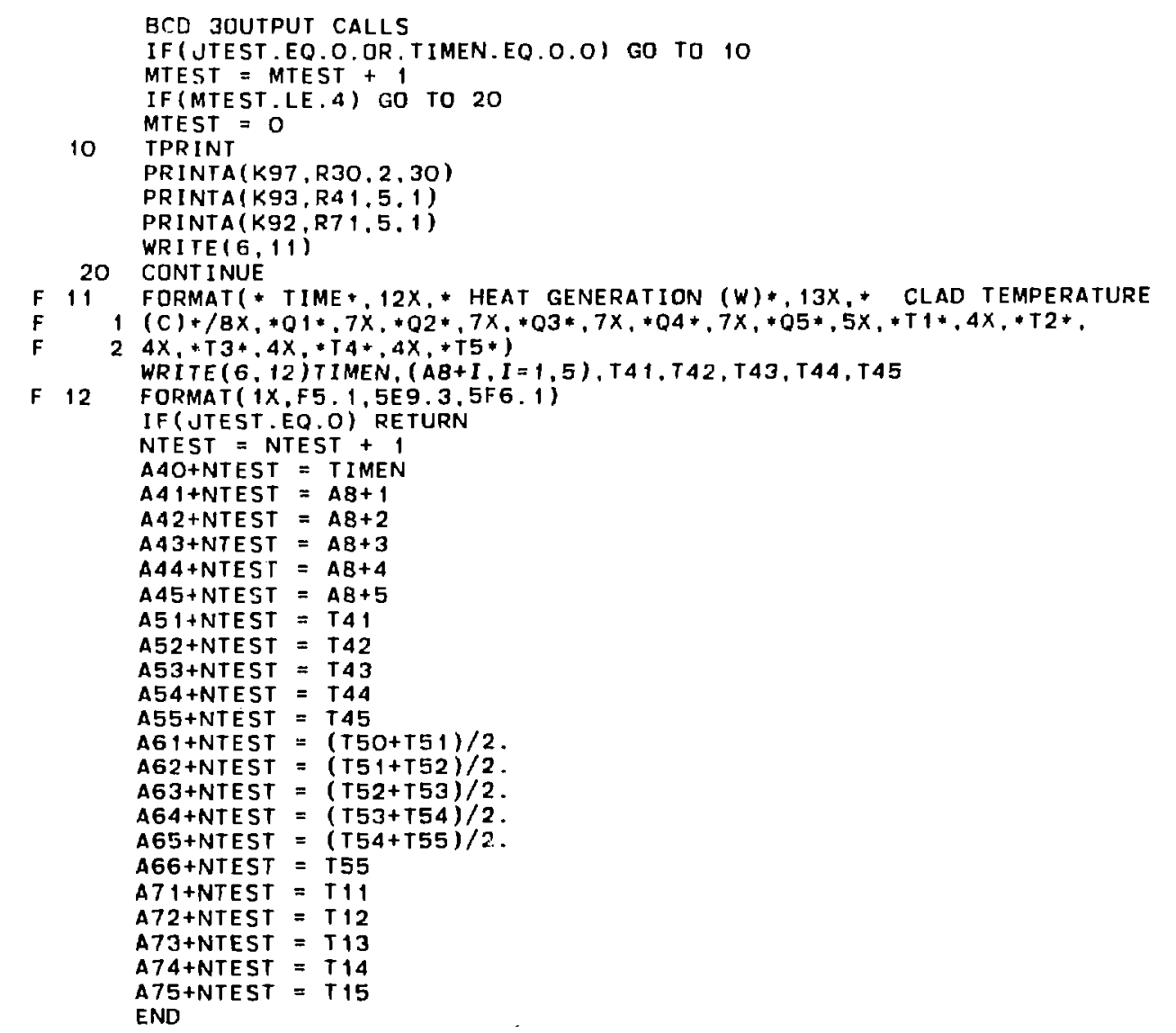

IF (JTEST.EQ.O OR TIMEN.EQ.O.O) GO TO 10 MTEST + 1 MTEST $=0$

PRINTA $(K 97, R 30,2,30)$

PRINTA $(K 93, R 41,5,1)$ , R7 $1,5,1$

TE (G, 1

11 FORMAT * TIME* $12 X$ * HEAT GENERATION $(w) * 13 X$ * CLAD TEMPERATURE

$(C)+/ B X * 01 * 7 X * 02 * 7 X * 03 *, 7 X * 04 * 7 X * 05 * 5 X * 11 *, 4 X * 12 *$

$4 X, * T 3 *, 4 X, * T 4 *, 4 X,+T 5 *$

TTEST NTEST +

$\triangle 41+$ NTEST $=A B+1$

NTEST $=A B+3$

END

A. 582

582
A. $\quad 582 \quad 583$

A. 584

A. $585 \quad 585$

A. $586 \quad 586$

A. $587 \quad 587$

A. $588 \quad 588$

A. $589 \quad 589$

A. $590 \quad 590$

A. $591 \quad 59$

A. $592 \quad 592$

A. $\quad 593 \quad 594 \quad 593$

A. $594 \quad 595$

A. $595 \quad 596$

A. $\quad 596 \quad 596$

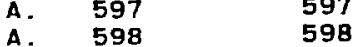

A. $599 \quad 599$

A. 600600

A. 601601

A. 602 ? 602

A. $603 \quad 604 \quad 603$

A. $605 \quad 605$

A. $605 \quad 605$

$\begin{array}{lll}\text { A. } & 606 & 606 \\ \text { A. } & 607 & 607\end{array}$

A. $608 \quad 608$

A. 609609

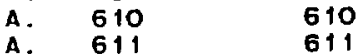

A. $612 \quad 612$

A. $613 \quad 613$

A. $614 \quad 614$

A. $615 \quad 615$

A. $616 \quad 616$

A. $617 \quad 617$

A. $619 \quad 619$

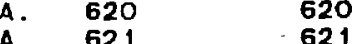

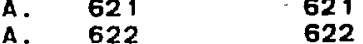

A. $618 \quad 618$ 
$\$$ TFLO, TFLOSS, DFUEL, DCLADO, DCLADI , PNOT, PORE. PINTF, GGAP)

THIS SUBROUTINE COMPUTES THE GAP CONDUCTANCE BETWEEN CLADOING ANO FUEL FOR BOTH SPHERE-PAC AND PELLET FUEL PINS.

AN "ADAPTEO" ROSS/STOUTE METHOD IS USED

AN "ADAPTEO" ROSS/STOUTE METHOD IS USED
WHERE THE DEPENDENCE OF CONDUCTANCE ON INTERFACE ORESSLRE IS A CURVE FIT BASED ON LIFE-AC COMPUTATIONS.

CONSTANTS FOR DG CLADOJNG

DATA AEC,BEC/2.01E+05, -79.29/

DATA AGC.BGC/7.86E+04,-3B.41/

DATA AAC,BAC/15.E-06.4.E-09/

CONSTANTS FOR CARBIDE FUEL

DATA AAF. BAF/9. IE-06, 1.4E-09/

DATA FOR PLENUM IC'S

DATA PPNOT, TPNOT/330000. $871 . /$

CALCULATE ELASTIC CONSTANTS

$T C L A D=0.5 *(T C I+T C O)$

$E C L A D=(A E C+B E C * T C L A D) * 1 . E+6$

$G C L A D=(A G C+B G C * T C L A D) * 1 . E+6$

$A L P H C=A A C+B A C * T C L A D$

GNUC $=\left(E C L A D /\left(2 .{ }^{*} G C L A D\right)\right)-1$

TFUEL $=0.5 *(T F I+T F O)$

EFUEL $=2.02 *(1 .-1.54 * P O R E) *(1 .-0.92 E-O 4 *($ TFUEL -25.$)) * 1 . E+11$ GFUEL $=0.78 *(1 .-1.54 * P O R E) *(1 .-0.92 E-O 4 *(T F U E L-25)) /.(1,-0.44 E-04 *$

$\$($ TFUEL-25.))*1.E+11

$A L P H F=A A F+B A F+T F U E L$

GNUF $=($ EFUEL $/(2, *$ GFUEL $))-1$

calculate change in plenum pressure

DELPP $=$ PPNOT * $(($ TFLO+273. $) /$ TPNOT - 1.0$)$

CALCULATE INTERFERANCE BASED ON NEW TEMPERATURES AND PLENUM PRESSURES

TICLAD $=0.5+(T C S S I+T C S S O)$

TIFUEL $=0.5+($ TFSSI +TFSSO)

RCI $=(D C L A D I / 2$. $) / 100$.

$R C I=(D C L A D I / 2) /$.100 .

THIKCL $=$ RCD - RC I

$R F=(D F U E L / 2) /$. 
DELTA $=((1+$ + GNUF $)+A L P H F \star R F *(T F U E L-T I F U E L)-A L P H C+R C I *(T C L A D-T I C L A D)$

\$) $-\mathrm{DCLPP}+(\mathrm{RCI} \cdot \mathrm{RCI} /(2,+\mathrm{ECLAD}+\mathrm{THIKCL}))$

Calculate interface pressure based on new delta

$D E N O M=R C I *(1, / E F U E L+(R C O * R C O+R C I+R C I) /(E C L A D *(R C D * R C O-R C I * R C I))$

$\$$ - GNUF/EFUEL + GNUIC/ECLAD)

PINTF $=$ DELTA /OENDM+PNOT

CALCULATE gaP CONDUCTANCE

IF (PINTF.LE.O.O) PINTF=0.O

GGAP $=1.005+5.218 *((P$ [NTF $* 1 . E-06) / 18.63) * 1.15$

RETURN

END

SUBROUT INE FILMITF 1, TF2, MCP, DCHAN, ACHAN, DCLAD, LEN, G1, G2, G3)

REAL MCP, LEN

$P I=3.14159$

ASURF = PI*DCLAD*LEN

$T F M=(T F 1+T F 2) / 2.0$

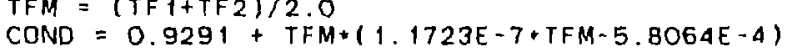

PECLET $=$ MCP $/ 2 .+D C H A N / A C H A N / C O N D$

HTC $=$ COND/DCHAN $*(0.012+\mathrm{PECLET}+.86+4.386)$

UA $=$ HTC + ASURF

IF(UA/MCP.GT.1, OE3) GO TO 10

$E F F=1 . O-E X P(-U A / M C P)$

$G 1=E F F+M C P$

$G 2=G 1$

$G 3=(1 . O-E F F) \cdot M C P$

RETURN

10 GI $=M C P$

$\mathrm{G2}=\mathrm{G1}$

$G 3=1 . O E-3$

RE TURN

END

SUBROUTINE CFUEL(TFUEL, LEN, DFUEL, PORO, CO, C1, C2, C3, C4

- CONDR, CONDAI

THIS SUBROUTINE CALCULATES FUEL CONDUCTANCE, AXIAL TivD RADIAL. AS A FUNCTION OF TEMPERATURE UNITS ARE W/CM-K FOR T IN KELVINS REAL LEN,KFUEL

TFIIEL $=$ TFUEL + $273 \cdot 15$

KFUEL $=(C O+T F U E L *(C 1+T F U E L+(C 2+T F U E L+(C 3+T F U E L+C 4))))$

IF (KFUEL GT O 15) KFUEL $=0.15$

CUNOR $=4 . O+K F U E L * 3.14159 * L E N$

CONDA $=$ KFUEL +3.14159+DFUEL+DFUEL/4.O/LEN

RETURN

BCD 3ENO DF DATA

RFL TO $127050 B$ REQUESTEO. OLO $F L=200000 B$. NEW FL $=200000 B$.

$\begin{array}{lll}\text { A. } & 675 & 675 \\ \text { A. } & 676 & 676 \\ \text { A. } & 677 & 677 \\ \text { A. } & 678 & 678 \\ \text { A. } & 679 & 679 \\ \text { A. } & 680 & 680 \\ \text { A. } & 681 & 681 \\ \text { A. } & 682 & 682 \\ \text { A. } & 683 & 683 \\ \text { A. } & 684 & 684 \\ \text { A. } & 685 & 685 \\ \text { A. } & 686 & 686 \\ \text { A. } & 687 & 687 \\ \text { A. } & 688 & 688 \\ \text { A. } & 689 & 689 \\ \text { A. } & 690 & 690 \\ \text { A. } & 691 & 691 \\ \text { A. } & 692 & 692 \\ \text { A. } & 693 & 693 \\ \text { A. } & 694 & 694 \\ \text { A. } & 695 & 695 \\ \text { A. } & 696 & 696 \\ \text { A. } & 697 & 697 \\ \text { A. } & 699 & 698 \\ \text { A. } & 699 & 699 \\ \text { A. } & 700 & 700 \\ \text { A. } & 701 & 701 \\ \text { A. } & 702 & 703 \\ \text { A. } & 703 & 702 \\ \text { A. } & 704 & 703 \\ \text { A. } & 705 & 704 \\ \text { A. } & 706 & 705 \\ \text { A. } & 707 & 706 \\ \text { A. } & 708 & 707 \\ \text { A. } & 709 & 708 \\ \text { A. } & 710 & 709 \\ \text { A. } & 711 & 710 \\ \text { A. } & 712 & 711 \\ \text { A. } & 713 & 712 \\ \text { A. } & 714 & 713 \\ \text { A. } & 715 & 714 \\ \text { A. } & 716 & 715 \\ \text { A. } & 717 & 716 \\ \text { A. } & 718 & 717 \\ \text { A. } & 719 & 718 \\ \text { A. } & 720 & 719 \\ \text { A. } & 721 & 720 \\ \text { A. } & 722 & 721 \\ \text { A. } & 723 & 722 \\ \text {. } & 723\end{array}$


THE INITIAL DATA VALUES AND FORTRAN PRDGRAMS ( AFTER EDITS) HAVE BEEN WRITTEN ON THE PROBLEM RESTART FILE -RSTRTO- AS PRDBLEM-STOOOO-.

CORE REQUIREMENTS FOR MITAS COMPILATION PHASE

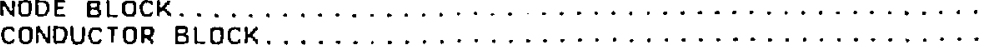

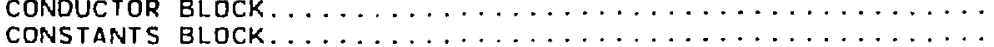

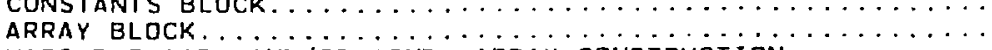

VARIAELE CAP. AND $/ O R$ COND. ARRAY CONSTRUCTION. . . . . . .

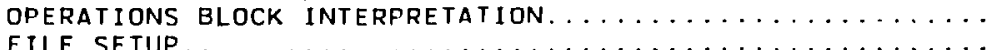

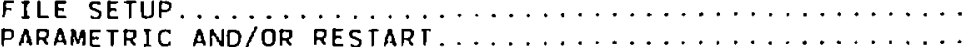

MINIMUM CORE REQUIRED FOR MITAS COMPILER

0116127 OCTAL

0116147 OCTAL

0116374 OCTAL

OT27130 DCTAL

DITJ7G DCTAL

0116376 DCTAL

0127050 DCTAL

OOOOOOO OCTAL

0127200 OCTAL

CP TIME CONSUMED IN MITAS COMPILATION PHASE

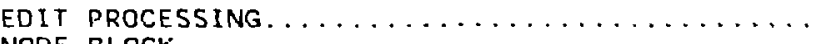

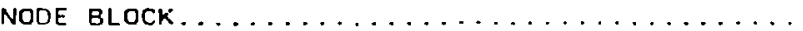

CONDUCTOR BLOCK. . . .

CONSTANT BLOCK $\ldots \ldots \ldots \ldots \ldots$

ARRAY BLOCK........................

VARIABLE CAP. AND/OR CONO

TOTAL TIME FOR DATA BLOCKS $\ldots \ldots \ldots \ldots \ldots$

TOTAL TIME FOR OPERATIONS BLOCK $\cdots \cdots \cdots \cdots$

TOTAL TIME FOR FILE SETUP.

TOTAL TIME FOR PARAMETRIC AND/OR RESTART ........

TOTAL CP TIME FOR MITAS COMPILATION PHASE.....
.229

322

.322
1.170

.052

2. 115 SEC

1.681 SEC

0.000 SEC

0.000 SEC

4.847 SEC

+++++ NORMAL TERMINATION OF MITAS COMPILER +++++ 